\title{
The balance of mitochondrial fission and fusion in cortical axons depends on the kinases SadA and SadB
}

Danila Di Meo ${ }^{1,2}$, Priyadarshini Ravindran ${ }^{1}$, Pratibha Dhumale ${ }^{1,2,3}$, Andreas W. Püschel ${ }^{1,2,4, *}$

${ }^{1}$ Institut für Molekulare Zellbiologie, Westfälische Wilhelms-Universität, Schloßplatz 5, D-48149 Münster, Germany

${ }^{2}$ Cells-in-Motion Interfaculty Center, University of Münster, D-48149 Münster, Germany

${ }^{3}$ Present address: Laboratory of Molecular and Cellular Cardiology, Institute of Clinical Research, University of Southern Denmark, Odense, Denmark

${ }^{4}$ Lead contact

*Correspondence: apuschel@uni-muenster.de 


\section{Summary}

Neurons are highly polarized cells that display characteristic differences in the organization of their organelles in axons and dendrites. Mitochondria are of particular importance for neuronal homeostasis due to their high metabolic demand. The kinases SadA and SadB (SadA/B) promote the formation of distinct axonal and dendritic extensions during the development of cortical and hippocampal neurons. Here, we show that SadA/B are required for the axon-specific dynamics of mitochondria. The interaction with Ankyrin B (AnkB) stimulates the activity of SadA/B that function as regulators of mitochondrial dynamics through the phosphorylation of Tau. Suppression of SadA/B or AnkB in cortical neurons induces the elongation of mitochondria by disrupting the balance of fission and fusion. The normal dynamics of axonal mitochondria could be restored by mild actin destabilization. Thus, the elongation after a loss of SadA/B results from an excessive stabilization of actin filaments and reduction of Drp1 recruitment to mitochondria.

\section{Introduction}

Neurons are highly polarized cells with morphologically and molecularly distinct axonal and somato-dendritic compartments that display characteristic differences in the organization of their organelles (Britt et al., 2016; Schelski and Bradke, 2017). Of particular importance for neuronal homeostasis are mitochondria. Defects in their morphology or dynamics lead to neurodevelopmental diseases and neurodegeneration (Baum and Gama, 2021). Mitochondria are dynamic organelles that are continuously remodeling their morphology by fission and fusion (Fenton et al., 2021; Moore, 2018). Mitochondrial dynamics is a finely tuned process that regulates their size and distribution and is essential for their function (Lee et al., 2018; Sheng and Cai, 2012). Fusion of the outer mitochondrial membrane (OMM) is mediated by Mitofusins while fission depends on the cytoplasmic GTPase Dynamin-related protein 1 (Drp1) that is recruited to the OMM by receptors like Mitochondrial fission factor (Mff) (Losón et al., 2013). Mitochondria are relatively short and uniform in size in axons while they are more elongated and display lower motility in the somato-dendritic compartment (Cagalinec et al., 2013; Chang et al., 2006; Lewis et al., 2018; Rangaraju et al., 2019; Saxton and Hollenbeck, 2012). Maintaining the size of axonal mitochondria depends on the coupling of fission and fusion and requires Mff (Lewis et al., 2018). Since Mff is present in both axons and dendrites the factors that are responsible for regulating the balance of fission and fusion and the size of mitochondria in the axon remain to be identified.

During the development of the nervous system, axons and dendrites arise from undifferentiated neurites that are extended by newborn unpolarized neurons (Lewis et al., 2013; Namba et al., 2015; Schelski and Bradke, 2017). Upon the establishment of neuronal polarity, one of these neurites is selected to differentiate as the axon, while the remaining neurites acquire the characteristics of dendrites. The kinases SadA and SadB (SadA/B, also known as Brsk2 and Brsk1) function redundantly to promote the formation of distinct axonal and dendritic extensions in 
cortical and hippocampal neurons (Barnes et al., 2007; Dhumale et al., 2018; Kishi et al., 2005; Müller et al., 2010; Shelly and Poo, 2011). They are also required at later stages for different aspects of neuronal differentiation like the central arborization of sensory axons as well as the maturation of synapses in the central and peripheral nervous system (Barnes et al., 2007; Kishi et al., 2005; Lilley et al., 2013; Shelly and Poo, 2011). They belong to the AMPK family of serine/threonine kinases and are activated by phosphorylation of Thr175 and Thr187, respectively, by the Lkb1/Strad complex (Barnes et al., 2007; Lizcano et al., 2004; Shelly and Poo, 2011; Veleva-Rotse et al., 2014). However, their regulation is cell-type specific. Not all functions of SadA/B depend on Lkb1 and other kinases can also activate them (Bright et al., 2008; Lilley et al., 2013).

A knockout of both Sada and Sadb severely reduces cortical axon tracts and the size of the cortex, which is not detectable in single knockouts (Dhumale et al., 2018; Kishi et al., 2005). The Sada;Sadb-deficient cortex shows extensive apoptosis of neurons and a reduction in the number of progenitors. Cultured hippocampal or cortical neurons from Sada ${ }^{-/} ; S^{-} \mathrm{Sadb}^{-/}$double knockout embryos fail to form distinct axonal and dendritic processes. Instead, a large proportion extends indeterminate neurites that are positive for both axonal and dendritic markers. The defect in axon formation has been linked to a function in the regulation of microtubule binding proteins (Barnes et al., 2007; Cavallini et al., 2013; Kishi et al., 2005). SadA/B and other AMPK-related kinases like Mark2 or Nuak1 phosphorylate the microtubule-associated protein Tau at Ser262 and Ser356, which attenuates the interaction with microtubules (Biernat et al., 1993; Drewes et al., 1995). However, how Tau regulates microtubule dynamics and contributes to the defects in neuronal polarity after the loss of SadA/B still remains incompletely understood. Tau is generally thought to stabilize microtubules, but recent results indicate that it promotes the elongation of labile microtubule domains without stabilizing them (Chang et al., 2021; Qiang et al., 2018). Tau oligomerizes to form condensations on microtubules that act as barriers for kinesins (Ambadipudi et al., 2017; Chang et al., 2021; Siahaan et al., 2019; Tan et al., 2019; Wegmann et al., 2018). The formation of condensates is promoted by its phosphorylation but does not require it. Tau hyperphosphorylation is a hallmark of Alzheimer's disease and has been linked to defects in the axonal transport of mitochondria (Chang et al., 2021).

In this study, we identified a novel function of SadA/B kinases as regulators of mitochondrial dynamics that is required for maintaining the typical size of mitochondria in cortical axons. Suppression of SadA/B leads to an elongation of mitochondria by disrupting the balance between fission and fusion. Our results show that the increased length of mitochondria after the loss of SadA/B results from an excessive stabilization of F-actin and an impairment of Drp1 recruitment to the OMM, due to the reduced phosphorylation of Tau. The scaffolding protein Ankyrin B (AnkB) functions upstream of SadA/B to stimulate their phosphorylation. 


\section{Results}

\section{AnkB interacts with SadA and B and stimulates their activation}

SadA/B are kept in an auto-inhibited state by the association of their $\mathrm{N}$-terminal kinase domain with the ubiquitin associated (UBA) domain and an auto-inhibitory sequence (AIS) at the C-terminus (Fig. 1A) (Ma et al., 2016; Wu et al., 2015). This auto-inhibition can be released by the interaction with scaffolding proteins (Hung et al., 2007; Ma et al., 2016; Wu et al., 2015). Active SadA and SadB, indicated by phosphorylation at Thr175 (SadA) and Thr186 (SadB), are restricted to the axonal compartment (Barnes et al., 2007; Dhumale et al., 2018). The scaffolding protein AnkB is a component of the submembranous cytoskeleton but also mediates bidirectional motility of organelles by linking them to the dynein/dynactin complex (Galiano et al., 2012; Lorenzo et al., 2014; Qu et al., 2016). To test if AnkB functions as a scaffold for SadA/B we co-expressed them in HEK 293T cells. Immunoprecipitation showed that AnkB interacts with both kinases (Fig. 1B). It binds to the $\mathrm{N}$-terminal fragment containing the kinase and UBA domains but not the C-terminal fragment of SadA and SadB (Fig. 1C).

To investigate whether the interaction with AnkB stimulates the phosphorylation of SadA/B we coexpressed increasing amounts of AnkB with SadA, SadB or non-phosphorylatable SadA-T175A and SadB-T186A in HEK 293T cells. In the absence of AnkB, only low levels of phosphoT175/T186-SadA/B were detectable by Western blot. With increasing expression of AnkB higher levels of phospho-T175/186-SadA/B were observed (Fig. 1D). No signals were detectable for the non-phosphorylatable SadA/B mutants (Fig. S1A). To analyze SadA/B activity we used the microtubule-binding protein Tau as a well-characterized substrate that is phosphorylated at Ser262 and Ser356 (Ercan et al., 2017; Kishi et al., 2005; Yoshida and Goedert, 2012). Wild type SadA/B or inactive SadA/B (SadA/B-KI) were co-expressed with Tau or non-phosphorylatable Tau-S262A, Tau-S356A or Tau-S262/356A in HEK 293T cells and analyzed by Western blot. Only low levels of Tau phosphorylation at Ser262 and Ser356 by endogenous kinases were detectable with SadA/B$\mathrm{KI}$. Overexpression of wild type SadA/B strongly increased Tau phosphorylation at both sites (Fig. S1B). Notably, co-expression of AnkB stimulates SadA/B activity and increased basal Tau

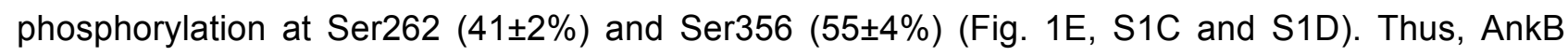
interacts with SadA/B and promotes their activation possibly by releasing the auto-inhibition to allow the phoshorylation by Lkb1 (Barnes et al., 2007; Lizcano et al., 2004).

\section{AnkB acts upstream of SadA/B}

Neurons from Sada ${ }^{-/} ;$Sadb $^{-/}$double knockout mice fail to form a distinct axon and instead extend several neurites that are relatively uniform in length and positive for both axonal and dendritic markers (Dhumale et al., 2018; Kishi et al., 2005; Müller et al., 2010). To study the function of SadA/B in cortical neurons but avoid the extensive loss of neurons by apoptosis in the Sada ${ }^{-/-}$ ;Sadb ${ }^{-/}$knockout cortex (Dhumale et al., 2018), we established knockdown vectors for SadA and 
SadB. The efficiency of the knockdown constructs was confirmed by Western blot after expression in HEK 293T cells (Fig. S2A). Cortical neurons from E18 rat embryos were transfected at 0 d.i.v. with knockdown vectors for SadA and SadB and analyzed at 3 d.i.v. by staining with antibodies for acetylated tubulin as axonal marker and MAP2 as dendritic marker (Fig. S2B). Transfected neurons were marked by expression of GFP from the knockdown vectors. The SadA/B knockdown induced the formation of indeterminate neurites that are positive for both axonal and dendritic markers (control: 13 $\pm 1 \%$; SadA/B RNAi: $44 \pm 1 \%$ ) as described before (Dhumale et al., 2018; Kishi et al., 2005; Müller et al., 2010). In addition, 33 $\pm 1 \%$ of the neurons extended supernumerary axons that are positive for acetylated tubulin but not MAP2 (Fig. S2B and S2C). This phenotype was rescued by co-expression of RNAi-resistant SadA/B, verifying the specificity of the knockdown constructs (Fig. S2D and S2E). After knockdown of SadA/B, the levels of phospho-S356-Tau detected by Western blot (Fig. S2F) and immunofluorescence were strongly reduced in cortical neurons at 3 d.i.v. (Fig. S2G and $\mathrm{S} 2 \mathrm{H}$ ).

In order to determine if AnkB is required for axon formation, cortical neurons were transfected with a knockdown vector at 0 d.i.v. The efficiency of the knockdown construct that targets the AnkB death domain shared by the 220 and $440 \mathrm{kDa}$ isoforms (Ayalon et al., 2011) was confirmed by Western blot (Fig. S2I). The knockdown of AnkB increased the number of neurons with indeterminate neurites (control: $6 \pm 0.4 \%$, AnkB RNAi: $37 \pm 1 \%$ ) and supernumerary axons (control: $14 \pm 1 \%$, AnkB RNAi: $29 \pm 1 \%$ ) similar to the knockdown of SadA/B (Fig. 2A and 2B). Expression of RNAi-resistant AnkB rescued this phenotype confirming the specificity of the knockdown construct (Fig. S2J). The expression of constitutively active SadA (SadAca) and SadB (SadBca) (Barnes et al., 2007; Lizcano et al., 2004; Müller et al., 2010) restored neuronal polarity after the knockdown of AnkB, confirming that it results from a reduced activation of SadA/B (Fig. 2C and 2D; AnkB RNAi: $35 \pm 2 \%$ indeterminate neurites, $28 \pm 1 \%$ supernumerary axons; AnkB RNAi+SadA/Bca: $17 \pm 2 \%$ indeterminate neurites; $17 \pm 2 \%$ supernumerary axons). Constitutively active SadA/B did not affect neuronal polarity in control neurons. These results indicate that AnkB acts upstream of SadA/B to stimulate their activity.

\section{The loss of SadA/B or AnkB increases the length of mitochondria}

AnkB localizes to intracellular membranes including mitochondria and lysosomes, while phosphoT175/186-SadA/B is restricted to the axon but not to a specific organelle (Fig. S3A) (Dhumale et al., 2018; Galiano et al., 2012; Lorenzo et al., 2014; Qu et al., 2016). To determine if they are present on mitochondria we labeled them by expression of mitoGFP and stained neurons at 3 d.i.v. with antibodies for AnkB and phospho-T175/186-SadA/B. Signals for AnkB were detectable on $72 \pm 2 \%$ of the mitochondria in axons (Fig. S3B and S3C), confirming previous results (Qu et al., 2016). AnkB-positive sites were analyzed for the presence of phosphorylated SadA/B (Fig. 3A and $3 B$ ). $38 \pm 1 \%$ of the mitochondria showed at least one site positive for both phosphorylated SadA/B 
and AnkB. Moreover, we observed that $53 \pm 3 \%$ of mitochondria showed at least one site of colocalization with both AnkB and Lamp1, as marker for lysosomes (Fig. 3C and 3D) and $48 \pm 2 \%$ with both phosphorylated SadA/B and Lamp1 (Fig. 3E and 3F). This colocalization suggests that the interaction of AnkB with SadA/B might stimulate their kinase activity at sites of contact between mitochondria and lysosomes.

Contacts with lysosomes mark sites of mitochondrial fission (Wong et al., 2018, 2019; Yoshimori et al., 2018). Therefore, we analyzed the morphology of mitoGFP-labeled mitochondria at 3 d.i.v. after knockdown of both SadA and SadB. The average length of mitochondria in controls is $1.6 \pm 0.1 \mu \mathrm{m}$ in axons and 3.6 $\pm 0.2 \mu \mathrm{m}$ in minor neurites (Fig. 4A, 4B and S4A). After knockdown of SadA and SadB, mitochondrial length was significantly increased to $3.2 \pm 0.2 \mu \mathrm{m}$ in indeterminate neurites and $3.0 \pm 0.1 \mu \mathrm{m}$ in supernumerary axons (Fig. 4A, 4B and S4B) that were analyzed separately. To exclude the possibility that the elongation of mitochondria after knockdown of SadA/B is a secondary consequence of the defect in the establishment of neuronal polarity, we transfected neurons at 1 d.i.v. instead of 0 d.i.v. (Fig. $4 \mathrm{C})$. The majority of neurons $(65 \pm 2 \%$ of the transfected H2B-mRFP-positive neurons) polarized normally and extended a single axon when transfected at this stage (Fig $S 4 C$ ). However, the length of axonal mitochondria was still significantly increased (Fig. 4D and S4D; control: 1.8 \pm 0.1 , SadA/B RNAi: $2.7 \pm 0.1$ ).

After knockdown of AnkB, the length of axonal mitochondria was increased to a similar extent (Fig. 4E, 4F and S4E; control: $1.6 \pm 0.03 \mu \mathrm{m}$, AnkB RNAi: $3.1 \pm 0.2 \mu \mathrm{m}$ (indeterminate neurites), $2.7 \pm 0.06$ $\mu \mathrm{m}$ (supernumerary axons)). The co-expression of constitutively active SadA/B reversed the elongation and completely restored normal mitochondrial length after AnkB knockdown (Fig. 4G, S4F and S4G; AnkB RNAi: 3.1 $\pm 0.07 \mu \mathrm{m}$ (indeterminate neurites), 2.8 $\pm 0.04 \mu \mathrm{m}$ (supernumerary axons); AnkB RNAi +SadA/Bca: $2.1 \pm 0.01 \mu \mathrm{m})$. Expression of SadA/Bca did not change the length of mitochondria in controls. Thus, Sad kinases are required for regulating the length of mitochondria downstream of AnkB independently of neuronal polarization.

\section{SadA/B are required to maintain the balance of mitochondrial fission and fusion}

In cortical axons, the rates of mitochondrial fission and fusion are balanced to maintain their morphology (Cagalinec et al., 2013; Lewis et al., 2018; Saxton and Hollenbeck, 2012). An imbalance in these rates changes mitochondrial length. To determine if the elongation of axonal mitochondria after the knockdown of SadA/B or AnkB results from impaired mitochondrial dynamics, we performed live cell imaging of mitoGFP- or mitoRFP-labeled mitochondria in the proximal and middle segment of cortical axons at 3 d.i.v. (Suppl. Video S1 and S2). Our analysis revealed a striking increase in mitochondrial motility in SadA/B knockdown neurons. The percentage of motile mitochondria was increased in both the anterograde (Fig. 5A and 5E; control: 16 $\pm 1 \%$; SadA/B RNAi: $40 \pm 5 \%$ (indeterminate neurites), $28 \pm 4 \%$ (supernumerary axons)) and 
retrograde direction (control: $16 \pm 2 \%$; SadA/B RNAi: $38 \pm 5 \%$ (indeterminate neurites), $33 \pm 4 \%$ (supernumerary axons)).

Next, we quantified the rate of mitochondrial fission and fusion as the number of events $/ \mathrm{min} / \mathrm{mitochondria.} \mathrm{In} \mathrm{control} \mathrm{neurons,} \mathrm{the} \mathrm{rate} \mathrm{of} \mathrm{fission}(0.011 \pm 0.001)$ was similar to the rate of fusion $(0.012 \pm 0.001)$. The knockdown of SadA/B resulted in a strong but unequal increase in the rates of both fission and fusion that disrupted their balance (Fig. 5B and 5F; indeterminate neurites: $0.034 \pm 0.003$ (fusion), $0.025 \pm 0.002$ (fission), supernumerary axons: $0.022 \pm 0.001$ (fission), $0.029 \pm 0.002$ (fusion)). This unequal increase leads to a reduction in the percentage of fission events as a fraction of all events (fission + fusion) compared to controls (Fig. S5A; control: $50 \pm 1 \%$ of all events; SadA/B RNAi: $41 \pm 1 \%$ (indeterminate neurites), $43 \pm 1 \%$ (supernumerary axons)).

Knockdown of AnkB resulted in a phenotype similar to that of SadA/B and induced an increase in both the number of motile mitochondria (Fig. $5 \mathrm{C}$ and $5 \mathrm{G}$ ) and the rate of mitochondrial fission and fusion (Fig 5D and 5H; AnkB RNAi: 0.039 \pm 0.002 (fusion) and $0.030 \pm 0.002$ (fission) in indeterminate neurites; $0.042 \pm 0.004$ (fusion) and $0.028 \pm 0.001$ (fission) in supernumerary axons). Similar to the SadA/B knockdown, the AnkB knockdown changed the balance of fission and fusion with a decrease in the percentage of fission events (Fig. S5B; control: $52 \pm 1 \%$; AnkB RNAi: $42 \pm 2 \%$ (indeterminate neurites); $38 \pm 2 \%$ (supernumerary axons)). Taken together, these results show that the knockdown of SadA/B or AnkB, while increasing mitochondrial motility and dynamics, disturbs the balance of fission and fusion by decreasing the percentage of fission events, which results in an elongation of mitochondria.

\section{A decrease in Drp1 recruitment affects mitochondrial dynamics}

Drp1 is a dynamin-related GTPase that oligomerizes on the OMM to induce fission (Cagalinec et al., 2013; Francy et al., 2015; Smirnova et al., 2001). Silencing of Drp1 results in a dramatic elongation of mitochondria. Therefore, we asked whether the relative reduction in mitochondrial fission after knockdown of SadA/B results from an impaired Drp1 recruitment to mitochondria. Staining with an anti-Drp1 antibody revealed Drp1 puncta both in the cytoplasm and on mitochondria at 3 d.i.v. (Fig. 6A) (Losón et al., 2013; Smirnova et al., 2001). The number of Drp1 puncta per mitochondria increases with their length in control neurons. While the average length of mitochondria is higher after knockdown of SadA/B, the number of Drp1 puncta per mitochondria was significantly reduced for every size class compared to controls (Fig. 6B). These results suggest that the knockdown of SadA/B impairs the recruitment of Drp1 to the OMM, which could explain the relative reduction in fission events.

To determine whether impaired recruitment of Drp1 is sufficient to alter mitochondrial dynamics we employed Drp1 with a mutation in the stalk domain (Drp1-R376E) that reduces the interaction with its receptor Mff (Otera et al., 2010). Drp1-R376E blocks Drp1 association with mitochondria and impairs mitochondrial fission. The expression of Drp1-R376E did not affect neuronal polarity (Fig. 
S6A) but resulted in an elongation of axonal mitochondria (Fig. 6C, 6D and S6B; Drp1 WT: 1.5 $\pm 0.03 \mu \mathrm{m}$, Drp1-R376E: $2.4 \pm 0.01 \mu \mathrm{m})$. Live cell imaging of Drp1-R376E-expressing neurons (Suppl. Video S3) showed a higher number of both mitochondrial fission and fusion events with a lower increase in fission than fusion (Fig. 6E and 6F; $0.0329 \pm 0.002$ (fission); $0.0204 \pm 0.001$ (fusion)) compared to wild type Drp1 (0.0130 0.0005 (fission); $0.0136 \pm 0.0003$ (fusion)). This unequal change results in a lower percentage of fission events compared to wild type Drp1 (Fig.

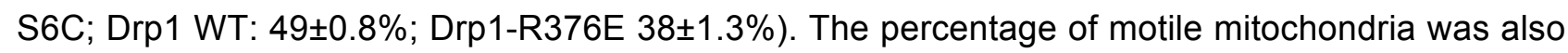
increased in both the antero- and retrograde direction by Drp1-R376E expression (Fig. 6G and $6 \mathrm{H})$. In summary, the expression of Drp1-R376E induced a change in mitochondrial dynamics that is very similar to that observed after the knockdown of SadA/B kinases.

\section{Tau regulates mitochondrial length downstream of SadA/B}

Overexpression of Tau results in defects in mitochondrial morphology due to an inhibition of Drp1 localization to mitochondria (DuBoff et al., 2012). In order to examine if SadA/B regulate mitochondrial size through the phosphorylation of Tau, we tested if expression of phosphomimetic Tau-S262D, Tau-S356D or Tau-S262/356D is able to rescue the defects in SadA/B knockdown neurons. Expression of phosphomimetic but not wild type Tau was able to restore neuronal morphology in SadA/B knockdown neurons, as predicted by previous results (Barnes et al., 2007; Kishi et al., 2005; Rodríguez-Asiain et al., 2011; Yoshida and Goedert, 2012) while it had no effect

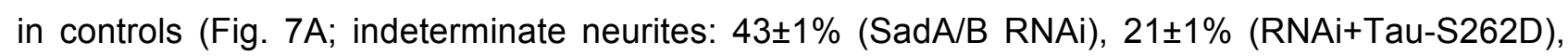
$21 \pm 1 \% \quad$ (RNAi+Tau-S356D), 12 $\pm 1 \%$ (RNAi+Tau-S262/356D); supernumerary axons: $33 \pm 2 \%$

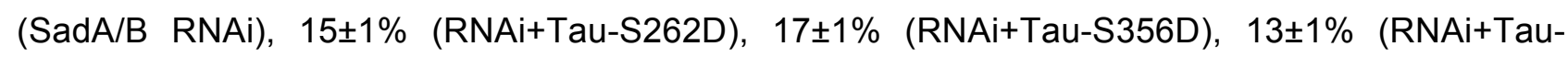
S262/356D). Notably, also mitochondrial length was fully normalized in SadA/B knockdown neurons by phosphomimetic but not wild type Tau (Fig. 7B, 7C, S7A and S7B; SadA/B RNAi 3.2 $\pm 0.1 \mu \mathrm{m}$ (indeterminate neurites), $2.8 \pm 0.2 \mu \mathrm{m}$ (supernumerary axons); RNAi+Tau-S262D:

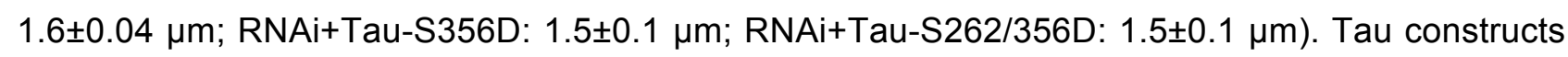
mimicking phosphorylation of a single Serine residue were already sufficient for a rescue. The restoration of mitochondrial length suggests that SadA/B kinases regulate the size of mitochondria by phosphorylating Tau.

It was previously shown that Tau interacts with and stabilizes F-actin (Cabrales Fontela et al., 2017; Elie et al., 2015; Fulga et al., 2007; He et al., 2009). Both excessive stabilization and complete depolymerization of actin filaments inhibits Drp1 translocation to mitochondria, resulting in mitochondrial elongation (DuBoff et al., 2012; Ji et al., 2015; Korobova et al., 2013, 2014). The phosphorylation of Ser262 and Ser356 in the repeat domain of Tau reduces its affinity for F-actin (Cabrales Fontela et al., 2017). Thus, decreased Tau phosphorylation might affect mitochondrial dynamics due to increased binding and excessive stabilization of actin filaments. To address this possibility, we tested if expression of Tau-L266E with a reduced affinity for F-actin can also rescue 
the knockdown of SadA/B (Cabrales Fontela et al., 2017). Expression of Tau-L266E restored neuronal polarity but did not affect its establishment in control neurons (Fig. 7D; indeterminate neurites: $40.3 \pm 1 \%$ (SadA/B RNAi), 15 $\pm 1 \%$ (RNAi+Tau-L266E); supernumerary axons: $33 \pm 1 \%$ (SadA/B RNAi), 12 $\pm 2 \%$ (RNAi+Tau-L266E)). Expression of Tau-L266E in SadA/B knockdown neurons significantly reduced mitochondrial length in neurons with a single axon to a value similar to controls (Fig. 7E, S7C and S7D; SadA/B RNAi: 2.9 $\pm 0.1 \mu \mathrm{m}$ (indeterminate neurites), $2.6 \pm 0.2 \mu \mathrm{m}$ (supernumerary axons); SadA/B RNAi+Tau-L266E: 1.6 $\pm 0.1 \mu \mathrm{m}$ ). Thus, the elongation of mitochondria upon knockdown of SadA/B can be rescued by expression of a Tau mutants that binds F-actin with decreased affinity.

\section{Actin destabilization rescues mitochondrial defects after knockdown of SadA/B}

To demonstrate that mitochondrial defects in SadA/B knockdown neurons arise from excessive Factin stability, we attempted to restore normal mitochondrial length by mildly destabilizing actin filaments with low doses of Latrunculin A (LatA). LatA sequesters actin monomers but also destabilizes actin filaments by severing and promoting depolymerization (Fujiwara et al., 2018). Cortical neurons were transfected with SadA/B knockdown vectors and treated with $1 \mathrm{nM}$ LatA. Unlike higher doses of LatA (Dupraz et al., 2019), low doses did not affect the establishment of polarity in control neurons. LatA also did not reduce the number of neurons with indeterminate neurites or supernumerary axons after knockdown of SadA/B (Fig. 8A). However, the elongation of mitochondria after loss of SadA/B could be fully reversed by LatA treatment for $17 \mathrm{~h}$ at 2 d.i.v. or for $5 \mathrm{~h}$ at 3 d.i.v. (Fig 8B, S8A, S8B and S8C; indeterminate neurites: $3 \pm 0.1 \mu \mathrm{m}$ (SadA/B RNAi), $2 \pm 0.1$ $\mu \mathrm{m}$ (SadA/B RNAi+17h LatA), $2.1 \pm 0.1 \mu \mathrm{m}$ (SadA/B RNAi+5h LatA); supernumerary axons: $2.9 \pm 0.2$ $\mu \mathrm{m}$ (SadA/B RNAi), 1.8 $\pm 0.1 \mu \mathrm{m}$ (SadA/B RNAi+17h LatA), 1.8 $\pm 0.1 \mu \mathrm{m}$ (SadA/B RNAi+5h LatA)).

To confirm that the reduction in mitochondrial length after actin destabilization is due to changes in the rates of fission and fusion, we analyzed mitochondrial motility and dynamics in neurons treated at 3 d.i.v. for 5 hours with $1 \mathrm{nM}$ LatA (Suppl. Video S4). The percentage of motile mitochondria was not significantly lower than in controls with vehicle after this short treatment (Fig. 8C and 8E). LatA does not alter the balance between mitochondrial fission and fusion in control neurons. However, the number of fission and fusion events was significantly reduced in SadA/B knockdown neurons treated with LatA (Fig. 8D and 8F; SadA/B RNAi: $0.045 \pm 0.002$ (fusion) and $0.034 \pm 0.002$ (fission) in indeterminate neurites; $0.028 \pm 0.0007$ (fission) and $0.036 \pm 0.0009$ (fusion) in supernumerary axons); SadA/B RNAi+LatA: $0.026 \pm 0.001$ (fission) and $0.017 \pm 0.0008$ (fusion) in indeterminate neurites; $0.022 \pm 0.001$ (fission) and $0.015 \pm 0.0007$ (fusion) in supernumerary axons). LatA changed the balance of fission and fusion and increased the percentage of fission events compared to controls (Fig. S8D; control: 50 $\pm 1 \%$; indeterminate neurites: $62 \pm 1 \%$; supernumerary axons: $60 \pm 1 \%)$. These results strongly suggest that the defects in mitochondrial dynamics after suppression of SadA/B are due to excessive actin stabilization. 


\section{Discussion}

As dynamic organelles, mitochondria continuously change their morphology and distribution while maintaining a relatively uniform length in axons through balanced rates of fission and fusion (van der Bliek et al., 2013; Cagalinec et al., 2013; Lewis et al., 2018; Seager et al., 2020; Tilokani et al., 2018). In this study we show that the kinases SadA and SadB function downstream of AnkB to regulate mitochondrial dynamics in cortical axons. We observed a striking increase in mitochondrial length after the loss of SadA/B due to alteration in the balance of fission and fusion. These changes result from excessive actin stabilization and decreased Drp1 localization to mitochondria due to reduced Tau phosphorylation. SadA/B are, therefore, required for the characteristic size of axonal mitochondria in cortical neurons. They join AMPK, Nuak1 and Mark2 that regulate various aspects of mitochondrial homeostasis (Courchet et al., 2013, 2018; Matenia et al., 2012; Toyama et al., 2016).

SadA and SadB are required for the differentiation of distinct axonal and dendritic extensions during neuronal development (Barnes et al., 2007; Dhumale et al., 2018; Kishi et al., 2005; Müller et al., 2010). The elongation of mitochondria after their loss could, therefore, arise as a secondary consequence of defects in axon formation. However, changes in mitochondrial dynamics were detected not only in indeterminate neurites but also in supernumerary axons formed after SadA/B knockdown. Moreover, the same mitochondrial elongation can be observed in normally polarized neurons when SadA/B were suppressed after axons are specified. Finally, mild actin destabilization rescued the imbalance in the rate of fission and fusion and normalized the length of mitochondria but did not restore neuronal polarity. Taken together, these results indicate that mitochondrial dynamics is directly affected by the loss of SadA/B independently of an effect on other axon-specific properties.

Live cell imaging revealed that the suppression of SadA/B disturbs the balance between fission and fusion by a shift towards a lower percentage of fission events. While both, fission and fusion events, were more frequent, this increase was unequal resulting in an elongation of mitochondria. The loss of SadA/B reduced the localization of Drp1 to mitochondria, which can explain the lower rate of fission. The recruitment and oligomerization of Drp1 to mitochondria is a critical step for fission and Drp1 deletion results in a striking elongation of mitochondria in axons (Berthet et al., 2014). Expression of the Drp1-R376E mutant with decreased affinity for the mitochondrial receptor Mff results in a similar phenotype as the knockdown of SadA/B. Thus, impairing the recruitment of Drp1 is sufficient to alter mitochondrial dynamics and length without affecting the polarization process. Fission and fusion of mitochondria do not occur independently of each other and are coordinated at contacts sites with the ER where the machinery for both processes is present (Abrisch et al., 2020; Guo et al., 2018). The reduced recruitment of Drp1 may, therefore, have consequences also for the fusion of mitochondria. 
It has been established before that Tau is a substrate for SadA/B (Barnes et al., 2007; Kishi et al., 2005; Rodríguez-Asiain et al., 2011; Yoshida and Goedert, 2012). Our experiments confirmed that Tau phosphorylation at Ser356 is reduced after their knockdown. Expression of phosphomimetic Tau was able to restore both neuronal polarity and normal mitochondrial length in SadA/B knockdown neurons, confirming that Tau is a major target for SadA/B in the developing nervous system required for both functions. Actin filaments promote Drp1 oligomerization and are essential for mitochondrial fission (Fenton et al., 2021; Moore, 2018). Tau binding was shown to excessively stabilize actin filaments in a model for tauopathy leading to impaired Drp1 localization (DuBoff et al., 2012). Tau phosphorylation at Ser262 and Ser356 attenuates the interaction with both microtubules and F-actin (Cabrales Fontela et al., 2017; Fulga et al., 2007). Therefore, a deficit in Tau phosphorylation could increase its binding and stabilization of actin filaments.

Depolymerization of actin by short-term treatment with high doses of Latrunculin $B$ reduces mitochondrial fission and increases the length of mitochondria (Korobova et al., 2013, 2014). However, treatment of SadA/B knockdown neurons with low doses of LatA to mildly destabilize actin filaments has the opposite effect and restores the relatively small and uniform size of mitochondria by increasing the percentage of fission events. These experiments provide direct evidence for the conclusion that the increase in mitochondrial length after knockdown of SadA/B arises from excessive F-actin stabilization. Actin filaments cycle through the mitochondrial network in HeLa cells by transiently assembling on subpopulations of mitochondria (Moore et al., 2016). This cycling of actin would be impaired by excessive stabilization by Tau since it requires a dynamic actin network, but it remains to be shown that it can be observed also in axons.

In addition to the elongation of mitochondria, a striking increase in the motility of mitochondria was observed after the knockdown of SadA/B that affected both antero- and retrograde transport. LatA treatment did not significantly alter mitochondrial motility in control or SadA/B knockdown neurons. The increased motility may, therefore, require higher LatA doses for a rescue or depend on an actin-independent mechanism. The transport of mitochondria along axonal microtubules is driven by kinesin- 1 and dynein (Fenton et al., 2021). Tau inhibits the transport by kinesin-1 by steric hindrance but has little effect on dynein (Chaudhary et al., 2018; Dixit et al., 2008; Ebneth et al., 2011; Monroy et al., 2018, 2020; Siahaan et al., 2019; Tan et al., 2019; Vershinin et al., 2007). However, an increased binding of Tau to microtubules due to a loss of SadA/B would be expected to decrease mitochondrial motility rather than increase it. Only a fraction of mitochondria is usually motile in axons, while the majority is stationary and anchored to microtubules by factors like Syntaphilin (Franker and Hoogenraad, 2013; Kang et al., 2008; Pathak et al., 2010). Syntaphilin inactivation results in a dramatic increase in their motility (Kang et al., 2008). Since the binding mode of Syntaphilin to the microtubule lattice remains to be characterized, it cannot be excluded that an increased localization of Tau to microtubules reduces the proportion of anchored mitochondria. The increase in the rate of motility and fusion suggests that SadA/B regulate several 
parameters of mitochondrial dynamics through additional mechanisms that remain to be elucidated in future studies.

We identified $A n k B$ as an upstream regulator of SadA/B that interacts with and stimulates their kinase activity as revealed by the phosphorylation of Tau. Knockdown of AnkB results in similar defects in neuronal morphology and mitochondrial size as a SadA/B knockdown and can be rescued by constitutively active SadA/B. AnkB associates with mitochondria and lysosomes in neurons (Lorenzo et al., 2014; Qu et al., 2016). Contact sites between those organelles mark the position of mitochondrial fission events (Wong et al., 2018, 2019; Yoshimori et al., 2018). A high percentage of mitochondria showed signals for AnkB that colocalized with phosphorylated SadA/B and both colocalized also with a lysosomal marker. The activation of SadA/B is, therefore, restricted to a limited number of sites along mitochondria, which may correspond to contacts between mitochondria and lysosomes based on their colocalization. Since phosphorylated SadA/B is not restricted to mitochondria, additional interaction partners are likely to regulate SadA/B activation at other sites (Hung et al., 2007; Ma et al., 2016; Wu et al., 2015; Yang et al., 2012). Our findings suggest that AnkB regulates mitochondrial motility and dynamics by promoting the activation of SadA/B in addition to its function as an adaptor for the dynein-dynactin motor complex (Lorenzo et al., 2014).

Taken together, our results suggest that $A n k B$ acts upstream of SadA/B in regulating mitochondrial motility and the balance of fission and fusion. SadA/B phosphorylate Tau and modulate mitochondrial dynamics by regulating the actin-dependent recruitment of Drp1 to mitochondria. These results identify a pathway in which SadA/B promote the acquisition of distinct properties of axonal mitochondria.

\section{Acknowledgments}

We thank Maria Wenning and Ina Kowsky for technical assistance and M. Bähler for plasmids. This work was supported by the Deutsche Forschungsgemeinschaft (DFG) through a grant to A.W.P. (PU-102-12), the Cells-in-Motion Cluster of Excellence (EXC 1003-CiM) and SFB 1348.

\section{Author Contributions}

A.P. conceived the study, D.D.M., P.R. and P.D. performed the experiments, D.D.M., P.R., P.D. and A.P. contributed to the experimental design and interpretation of the results, and D.D.M., P.R. and A.P. wrote the manuscript.

\section{Declaration of Interests}

The authors declare no competing interests. 


\section{Figure legends}

Figure 1. Ankyrin B interacts with SadA/B and stimulates their activation

(A) Schematic representation of SadA and SadB showing the domains used for interaction studies. (B and C) HA-AnkB and GFP-SadA, GFP-SadB (B) or the indicated fragments of SadA and SadB (C) were coexpressed in HEK 293T cells, immunoprecipitated and analyzed by Western blot with the indicated antibodies.

(D) Wild type SadA and SadB were coexpressed with increasing amounts of HA-AnkB in HEK 293T cells (SadA/B:AnkB expression vector ratio: 1:5,1:10 and 1:15). SadA/B phosphorylation was analyzed by Western blot with the indicated antibodies.

(E) Wild type (SadA/B WT) or inactive SadA and SadB (SadA/B-KI), HA-AnkB and mRuby2-Tau were co-expressed in HEK 293 T cells as indicated. Tau phosphorylation was analyzed by Western blot with the indicated antibodies.

See also Figures S1.

\section{Figure 2. AnkB acts upstream of SadA/B}

(A) Cortical neurons were transfected with control or AnkB knockdown vectors expressing GFP (AnkB RNAi, green) and stained with anti-acetylated tubulin (light gray) and -Map2 (red) antibodies. Scale bars: $20 \mu \mathrm{m}$.

(B) The percentage of unpolarized neurons without an axon (0), polarized neurons with a single axon (Axon), neurons with indeterminate neurites (Indet) and supernumerary axons (Spn) is shown for (A) (unpaired Student's t-test; $n=4$ independent experiments).

(C) Cortical neurons were transfected with control or AnkB knockdown vectors expressing H2BmRFP (pseudo-colored blue) and GFP (control) or constitutively active SadA and SadB (SadA/Bca; green). Neurons were stained with anti-acetylated tubulin (magenta) and -Map2 (light gray) antibodies. Scale bars: $20 \mu \mathrm{m}$.

(D) The percentage of unpolarized neurons without an axon (0), polarized neurons with a single axon (Axon), neurons with indeterminate neurites (Indet) and supernumerary axons (Spn) is shown for (C) (unpaired Student's t-test; $n=3$ independent experiments).

Values are means \pm SEM. See also Figure S2.

\section{Figure 3. AnkB, phosphorylated SadA/B and Lamp1 colocalize at axonal mitochondria}

(A) Neurons expressing mitoGFP (green) were stained with antibodies against phospho-T175/186SadA/B (magenta) and AnkB (blue).

(B) The percentage of mitochondria showing sites positive for both phospho-T175/186-SadA/B and AnkB is shown for $(A)$.

(C) Neurons expressing mitoGFP (green) were stained with antibodies against Lamp1 (magenta) and AnkB (blue). 
(D) The percentage of mitochondria showing sites positive for both Lamp1 and AnkB is shown for (C).

(E) Neurons expressing mitoGFP (green) were stained with antibodies against Lamp1 (magenta) and phospho-T175/186-SadA/B (blue).

(F) The percentage of mitochondria with sites positive for both LAMP1 and phospho-T175/186SadA/B is shown for $(E)$.

Higher magnification Airyscan images are shown in the lower panels. White arrows indicate sites of colocalization at mitochondria. Scale bars: $5 \mu \mathrm{m}$ (upper panels), $2 \mu \mathrm{m}$ (lower panels) ( $\mathrm{N}=90$ neurons from $n=4$ independent experiments). Values are means \pm SEM. See also Figure S3.

\section{Figure 4. The loss of SadA/B or AnkB increases the length of mitochondria}

(A) Cortical neurons were transfected with a vector for mitoGFP (green) and control or SadA/B knockdown vectors expressing H2B-mRFP (magenta). Higher magnification Airyscan images are shown in the lower panels. Scale bars: $20 \mu \mathrm{m}$ (upper panels), $2 \mu \mathrm{m}$ (lower panels).

(B) The average mitochondrial length is shown for $(A)$ (Indet: indeterminate neurites, Spn: supernumerary axons; one-way ANOVA followed by Dunnett's test; $N=125$ neurons for each condition from $n=5$ independent experiments).

(C) Cortical neurons were transfected at 1 d.i.v. and analyzed at 4 d.i.v. as in (A). Scale bars: $20 \mu \mathrm{m}$ (upper panels), $2 \mu \mathrm{m}$ (lower panels)

(D) The average mitochondrial length is shown for (C) (unpaired Student's t-test; $N=100$ neurons for each condition from $n=4$ independent experiments)

(E) Cortical neurons were transfected with a vector for mitoRFP (magenta) and control or AnkB knockdown vectors expressing GFP (green). Scale bars: $20 \mu \mathrm{m}$ (upper panels), $2 \mu \mathrm{m}$ (lower panels).

(F) The average mitochondrial length is shown for $(E)$ (Indet: indeterminate neurites, Spn: supernumerary axons; one-way ANOVA followed by Dunnett's test; $N=100$ neurons for each condition from $n=4$ independent experiments).

(G) Quantification of average mitochondrial length in cortical neurons transfected with a vector for mitoRFP, control or AnkB knockdown vectors and GFP (control) or constitutively active SadA and SadB (SadA/Bca) (In: indeterminate neurites; Sn: supernumerary axon; one-way ANOVA followed by Tukey's test; $n=3$ independent experiments).

Values are means \pm SEM. See also Figure S4.

Figure 5. SadA/B are required to maintain the balance of fission and fusion

( $A$ and $C$ ) Cortical neurons were transfected with a vector for mitoGFP ( $A$ and $B$ ) or mitoRFP (C and $D$ ) and control, SadA/B (A and B) or AnkB (C and D) knockdown vectors. Mitochondrial 
dynamics was analyzed by live cell imaging. Kymographs show anterograde (magenta) and retrograde (grey) transport of mitochondria in control (Axon) or knockdown neurons.

( $B$ and $D$ ) Selected frames from time-lapse videos show mitochondrial fission and fusion events from control (Axon), SadA/B (B) or AnkB (D) knockdown neurons. Red arrows mark fission or fusion events. Scale bars: $5 \mu \mathrm{m}$.

( $E$ and $G$ ) The percentage of anterogradely (Ant) and retrogradely (Ret) moving axonal mitochondria after knockdown of SadA/B $(E)$ or AnkB $(G)$ is shown $(N=22(E)$ and $12(G)$ neurons for each condition from $n=6(E)$ and $n=3(G)$ independent experiments)

$(F$ and $H)$ Fission and fusion were quantified after knockdown of SadA/B $(F)$ or AnkB $(H)$ as the number of events/min/mitochondria $(\mathrm{N}=22(\mathrm{~F})$ and $12(\mathrm{H})$ neurons for each condition from $n=6(F)$ and $n=3(H)$ independent experiments) (Indet: indeterminate neurites, Spn: supernumerary axons; one-way ANOVA followed by Dunnett's test was done or all experiments).

Values are means \pm SEM. See also Figures S5 and Videos S1 and S2.

\section{Figure 6. A decrease in Drp1 recruitment affects mitochondrial dynamics}

(A) Cortical neurons were transfected with a vector for mitoRFP (red) and control or SadA/B knockdown vectors and stained with an anti-Drp1 antibody (white). Scale bars: $5 \mu \mathrm{m}$.

(B) The number of Drp1 puncta localizing to mitochondria was assessed by Manders' colocalization coefficient-based analysis and is shown for different size classes of mitochondria (Indet: indeterminate neurites, Spn: supernumerary axons; one-way ANOVA followed by Tukey's test; $N=120$ neurons for each condition for $n=4$ independent experiments). The total number of mitochondria for each size class is shown below.

(C) Cortical neurons were transfected with vectors for mitoGFP and wild type Drp1 (Drp1 WT) or Drp1-R376E. Scale bars: $20 \mu \mathrm{m}$ (upper panels), $2 \mu \mathrm{m}$ (lower panels).

(D) The average mitochondrial length is shown for (C) (unpaired Student's t-test; $N=75$ neurons for each condition from $n=3$ independent experiments).

(E) Selected frames from time-lapse imaging show mitochondrial fission and fusion events from cortical neurons transfected as in (C). Red arrows mark fission or fusion events. Scale bars: $5 \mu \mathrm{m}$.

(F) Fission and fusion events were quantified from $(E)$ as the number of events/min/mitochondria (unpaired Student's t-test; $\mathrm{n}=4$ independent experiments).

(G) Kymographs show anterograde (magenta) and retrograde (light gray) axonal transport of mitoGFP-labelled mitochondria in neurons expressing wild type Drp1 (WT) or Drp1-R376E.

$(\mathrm{H})$ The percentage of anterogradely (Ant) and retrogradely (Ret) moving axonal mitochondria is shown for (G) (unpaired Student's t-test; $n=4$ independent experiments).

Values are means \pm SEM. See also Figures S6 and Video S3. 


\section{Figure 7. Tau regulates mitochondrial length downstream of SadA/B}

(A) Cortical neurons were transfected with control or SadA/B knockdown vectors and vectors for wild type or phosphomimetic Tau as indicated. The percentage of unpolarized neurons without an axon (0), polarized neurons with a single axon (Axon), neurons with indeterminate neurites (Indet) and supernumerary axons (Spn) is shown (one-way ANOVA followed by Tukey's test; $n=3$ independent experiments).

(B) The average mitochondrial length is shown for (A) (Indet: indeterminate neurites, Spn: supernumerary axons; one-way ANOVA followed by Dunnett's test; $n=3$ independent experiments)

(C) Higher magnification Airyscan images of mitoGFP (green) and wild type or phosphomimetic Tau (magenta) are shown for (B). Scale bars: $2 \mu \mathrm{m}$

(D) Cortical neurons were transfected with control or SadA/B knockdown vectors and a vector for wild type Tau or Tau-L266E. The percentage of unpolarized neurons without an axon (0), polarized neurons with a single axon (Axon), neurons with indeterminate neurites (Indet) and supernumerary axons (Spn) is shown (unpaired Student's t-test; $n=3$ independent experiments).

(E) The average mitochondrial length is shown for (D) (In: indeterminate neurites, Sn: supernumerary axons; one-way ANOVA followed by Tukey's test; $n=3$ independent experiments). Values are means \pm SEM. See also Figures $S 7$.

\section{Figure 8. Actin destabilization rescues mitochondrial defects after SadA/B knockdown}

(A) Cortical neurons were transfected with control or SadA/B knockdown vectors and treated with vehicle (DMSO) for $17 \mathrm{~h}$ or $1 \mathrm{nM}$ LatA for $17 \mathrm{~h}$ and $5 \mathrm{~h}$ as indicated. The percentage of unpolarized neurons without an axon (0), polarized neurons with a single axon (Axon), neurons with indeterminate neurites (Indet) and supernumerary axons (Spn) is shown (one-way ANOVA followed by Tukey's test; $n=3$ independent experiments).

(B) The average mitochondrial length is shown for (A) (In: indeterminate neurites. Sn: supernumerary axons; one-way ANOVA followed by Dunnett's test; $n=3$ independent experiments).

(C) Cortical neurons were transfected with control or SadA/B knockdown vectors and treated with vehicle (DMSO) or $1 \mathrm{nM}$ LatA for $5 \mathrm{~h}$. Mitochondrial dynamics was analyzed by live cell imaging. Kymographs show anterograde (magenta) and retrograde (light gray) axonal transport of mitochondria in control (Axon) or SadA/B knockdown neurons treated with 1nM LatA for $5 \mathrm{~h}$.

(D) Selected frames from time-lapse imaging show mitochondrial fission and fusion events from control (Axon) and SadA/B knockdown neurons treated with 1nM LatA for 5h (Indet: indeterminate neurites; Spn: supernumerary axons). Red arrows mark fission or fusion events. Scale bars: $5 \mu m$.

(E) The percentage of anterogradely (Ant) and retrogradely (Ret) moving axonal mitochondria are shown for (C) (one-way ANOVA followed by Tukey's test; $n=4$ independent experiments). 
bioRxiv preprint doi: https://doi.org/10.1101/2021.07.18.452621; this version posted July 19, 2021. The copyright holder for this preprint (which was not certified by peer review) is the author/funder. All rights reserved. No reuse allowed without permission.

(F) Fission and fusion events were quantified from (D) as the number of events/min/mitochondria (Indet: indeterminate neurites, Spn: supernumerary axons; one-way ANOVA followed by Tukey's test; $n=4$ independent experiments).

Values are means \pm SEM. See also Figures S8 and Video S4. 


\section{STAR Methods}

\section{LEAD CONTACT AND MATERIALS AVAILABILITY}

Further information and requests for resources and reagents should be directed to and will be fulfilled by the corresponding author, Dr. Andreas Püschel (apuschel@uni-muenster.de). All unique/stable reagents generated in this study are available from the corresponding author without restriction.

\section{EXPERIMENTAL MODEL AND SUBJECT DETAILS}

All animal protocols were performed in accordance with the guidelines of the North RhineWestphalia State Environment Agency (Landesamt für Natur, Umwelt und Verbraucherschutz (LANUV)). Rats were maintained at the animal facility of the Institute for Molecular Cell Biology (University of Münster) under standard housing conditions at four to five per cage with a $12 \mathrm{~h}$ light/dark cycle (lights on from 07:00 to $19: 00 \mathrm{~h}$ ) at constant temperature $\left(23^{\circ} \mathrm{C}\right)$ with continuous access to food and water. Timed-pregnant rats were set up in house. Pregnant rats were anesthetized by exposure to isoflurane followed by decapitation and primary cultures prepared from E18 embryos

\section{METHOD DETAILS}

\section{Neuronal Cell Culture and Transfection}

Primary cortical neurons were obtained from embryonic day 18 (E 18) rat embryos and transfected by calcium phosphate co-precipitation. In brief, cortices were dissected, collected in HBSS (Invitrogen) and digested in trypsin (0.25\% Trypsin/EDTA, Invitrogen) for $8 \mathrm{~min}$. Digested tissue was mechanically dissociated in DMEM (1X DMEM (Invitrogen), 2mM glutamine, 50U/mL gentamycin, $10 \%$ fetal calf serum (FCS)) and passed through a cell strainer (40 $\mu \mathrm{m}$ pore size, Sarstedt). Dissociated neurons were seeded onto poly-L-ornithine coated (10 $\mu \mathrm{g} / \mathrm{ml}$, SigmaAldrich) coverslips at a density of 35,000 cells $/ \mathrm{cm}^{2}$ for immunofluorescence staining on poly-L-ornithine coated $35 \mathrm{~mm}$ dish ( $\mu$-Dish Quad, Ibidi) at a density of 150,000 cells $/ \mathrm{cm}^{2}$ for live cell imaging. Neurons were allowed to attach in the presence of DMEM for $2 \mathrm{~h}$. The medium was then replaced by Neurobasal medium ((1x NBM (Invitrogen), 2mM glutamine, 50U/mL gentamycin, 2\% B27 (v/v)) or BrightCell ${ }^{\mathrm{TM}}$ NEUMO photostable medium (1X NEUMO (Merck Millipore), 4\% SOS, $4 \mathrm{mg} / \mathrm{ml}$ human recombinant insulin, $2 \mathrm{mM}$ glutamine, $50 \mathrm{U} / \mathrm{mL}$ gentamycin) for staining or live cell imaging, respectively. For transfection, the culture medium was replaced $3 \mathrm{~h}$ after plating by Opti-MEM (Invitrogen) before the DNA mixture was added. After incubation for 45 min at $37^{\circ} \mathrm{C}$ at $5 \% \mathrm{CO}_{2}$, neurons were washed for $15 \mathrm{~min}$ with $1 \mathrm{ml}$ of Opti-MEM, which had been pre-incubated at $37^{\circ} \mathrm{C}$ at 
$10 \% \mathrm{CO}_{2}$, and the conditioned Neurobasal medium was added back to the cells. Cortical neurons were transfected at 0 d.i.v. and fixed at 3 d.i.v. if not indicated otherwise.

For analysis by Western blot, primary cortical neurons were transfected by nucleofection using Nucleofector ${ }^{\mathrm{TM}}$ /I (Amaxa) and the Ingenio ${ }^{\circledR}$ Electroporation kit (Mirus Bio LLC). For one well of a 6-well plate, $1.5 \times 10^{6}$ cells were resuspended in $100 \mu \mathrm{L}$ Ingenio $\circledast$ electroporation solution with 3 $\mu \mathrm{g}$ of plasmid DNA and nucleofection was performed using the program 0-003. For analysis of phospho-S356-Tau levels, neurons were lysed in RIPA buffer $72 \mathrm{~h}$ after seeding.

\section{Plasmids}

The following plasmids were obtained from Addgene: pAnkyrinB-2HA (\#31057); pLV-RFP (\#26001); pmRuby2-MAPTau-C-10 (\#55904); pmCh-Drp1 (\#49152); psfGFP-C1 (\#54579). Red FP Vector-Mitochondrion (mitoRFP (\#558722)) was purchased from BD Pharmingen. MitochondrionEGFP (mitoGFP) was a gift from Martin Bähler (Oeding et al., 2018).

The expression vectors for EGFP-SadA-WT, EGFP-SadB-WT, EGFP-SadA-KI (S179D), EGFPSadB-KI (S191D), EGFP-SadAca (T175E) and EGFP-SadBca (T187E) have been described before (Müller et al., 2010).

The constructs expressing the Kinase-UBA (SadA: 19-342 aa; SadB: 33-358 aa) or AIS-KA1 (SadA: 343-636 aa; SadB: 359-715 aa) (Fig. 1A) domains of SadA/B were prepared from fulllength mouse SadA and SadB using the primers described in Table S1. The PCR products were cloned into the psfGFP-C1.

Loss of function experiments were performed with miRNAs generated using the BLOCK-iT Pol II miRNA Expression Vector Kit (Invitrogen) (Table S1). Target sequences were cloned into the pcDNA6.2-GW/EmGFP-miR expression plasmid following the manufacturer's instructions. To generate pcDNA6.2-GW/H2B-mRFP-miR, H2B-mRFP was excised from pLV-RFP with Dral/Sall and inserted into pcDNA6.2-GW/EmGFP to replace the EmGFP sequence.

Single point mutations were generated by site directed mutagenesis using Phusion High-Fidelity DNA Polymerase (Thermo Fisher) (Table S2). Tau mutants (Tau-S262D, Tau-356D, Tau262/356D, Tau-S262A, Tau-S356A, Tau-S262/356A and Tau-L266E) were generated from pmRuby2-MAPTau-C-10. The Drp1-R376E mutant was generated from pmCh-Drp1. Nonphosphorylatable SadA and SadB mutants (SadA-T175A, SadB-T186A) were generated from pEGFP-SadA and pEGFP-SadB. All the primers were purchased from Metabion.

\section{Immunofluorescence staining}

Neuronal cultures were fixed with $4 \%$ paraformaldehyde and $4 \%$ sucrose for $15 \mathrm{~min}$ at $37^{\circ} \mathrm{C}$ and quenched in $50 \mathrm{mM}$ ammonium chloride at room temperature (RT) for $10 \mathrm{~min}$. After several washes with Phosphate-Buffered Saline (PBS), the cells were permeabilized with $0.1 \%$ Triton X- 
100 for $3 \mathrm{~min}$ and treated with blocking solution (2\% normal goat serum, 2\% bovine serum albumin and $0.2 \%$ fish gelatine in PBS) for $1 \mathrm{~h}$ at RT. Fixed cells were incubated overnight with the primary antibodies (listed in the key resources table) at $4^{\circ} \mathrm{C}$. Cells were washed with PBS and incubated with the appropriate Alexa-Fluor conjugated secondary antibody (Thermo Fisher) at RT for $2 \mathrm{~h}$ and mounted using Mowiol (SigmaAldrich). Antibodies were diluted in 10\% blocking solution.

\section{Transfection of HEK 293T cells and Western blot}

HEK293T cells were transfected using the calcium phosphate co-precipitation method. Transfected HEK293T cells were harvested in ice-cold PBS and lysed at $4^{\circ} \mathrm{C}$ for $1 \mathrm{~h}$ (Lysis buffer: Tris/ $\mathrm{HCl} 50$ mM, pH 7,4, NaCl 150 mM, DTT 1 mM, MgCl2 1,5 mM, EDTA 4 mM, glycerol 10\% (v/v), Triton X$1001 \%(\mathrm{v} / \mathrm{v})$, complete protease inhibitor (Roche), tyrosine and serine/threonine phosphatase inhibitors (Sigma). After addition of $5 x$ Laemmli buffer and boiling at $95^{\circ} \mathrm{C}$, proteins were separated by SDS-polyacrylamide gel electrophoresis and transferred to nitrocellulose membranes. Nonspecific binding was blocked at RT with $5 \%$ non-fat dry milk or $3 \%$ fish gelatin (for detection of phosphorylated proteins, SigmaAldrich) in Tris-Buffered Saline and 0.1\% Tween20 (TBS-T) for $1 \mathrm{~h}$. The membrane was incubated overnight with primary antibodies diluted in blocking solution at $4^{\circ} \mathrm{C}$. After several washes with TBS-T, membranes were incubated with HRP-coupled secondary antibodies (Jackson ImmunoResearch Labs) for $2 \mathrm{~h}$ at RT. Peroxidase activity was visualized by the enhanced chemiluminescence detection system (Interchim) using the ChemiDocTM MP imaging system (Bio-Rad).

\section{Immunoprecipitation with anti-GFP nanobodies}

For co-immunoprecipitation experiments, a GST-tagged anti-GFP nanobody was expressed in E. coli BL21 cells as described (Katoh et al., 2015). The anti-GFP nanobody was coupled to Glutathione Sepharose ${ }^{\mathrm{TM}} 4 \mathrm{~B}$ beads (GE Healthcare) and incubated with lysates from transfected HEK $293 \mathrm{~T}$ cells for $1 \mathrm{~h}$ at $4^{\circ} \mathrm{C}$. The beads were washed three times $(10$ minutes each with Tris/ $\mathrm{HCl}$ pH 7.450 mM, NaCl 250 mM, DTT 1 mM, MgCl2 1.5 mM, EDTA 4 mM, glycerol 10\% (v/v), Triton $\mathrm{X}-1000.1 \%(\mathrm{v} / \mathrm{v})$, complete protease inhibitor). Bound proteins were eluted using $2 \mathrm{x}$ Laemmli buffer and analyzed by western blotting.

\section{Image acquisition and analysis}

Images were acquired on a Zeiss LSM 800 Airyscan laser scanning confocal microscope with 405, 488, 568, and $647 \mathrm{~nm}$ laser lines and processed using the Zeiss ZEN 2.3 (blue edition) software (Carl Zeiss Microlmaging, Jena, Germany). Neuronal and mitochondrial morphology was analyzed in fixed neurons using a Plan-Apochromat 40x/1,3 Oil DIC M27 objective. Co-localization studies 
were performed with a Plan-Apochromat 40x/1,3 Oil DIC M27 objective using images acquired with the Airyscan detector (Carl Zeiss Microlmaging, Jena, Germany). Time-lapse imaging for mitochondrial dynamics analysis was performed in a $\mathrm{CO}_{2}$ regulated incubation chamber maintained at $37^{\circ} \mathrm{C}$ (PeCon, Erbach). Images were acquired at a frame rate of one scan every 10 seconds for 25-45 minutes. Images were analyzed using ImageJ (NIH).

\section{QUANTIFICATION AND STATISTICAL ANALYSIS}

\section{Quantification}

Neuronal polarity was analyzed at 3 d.i.v as described previously (Dhumale et al., 2018). Neurons showing acetylated-tubulin-negative neurites that were positive for MAP2 were categorized as unpolarized and neurons with a single long and acetylated-tubulin-positive neurite as normally polarized (Witte et al., 2008). Neurons with several long and acetylated-tubulin-positive neurites showing accumulation of acetylated-tubulin were counted as neurons with supernumerary axons and those with several neurites of comparable length and positive for both, axonal (acetylatedtubulin) and dendritic markers (Map2), as neurons with indeterminate neurites. At least 150 cells per condition were counted for each experiment.

Mitochondrial length was quantified in selected ROls (scanning zoom: 1.2-1.5X) with resolvable individual mitochondria. User-defined thresholds for pixel intensity were used on maximum intensity projections of mitoGFP or mitoRFP stacks to identify measurable mitochondria and make binary images. The length of single mitochondria was then obtained by using the analyze particles tool. The analysis was performed on equal numbers of neurons for each condition in all experiments. The number of mitochondria moving along the axon was quantified from color-coded kymographs that were generated using the macro toolset KymographClear in ImageJ (Mangeol et al., 2016). The rate of fusion and fission was quantified by frame-by-frame manual analysis. For the analysis of colocalization, Airyscan images were pre-processed by filtering and adjusting the fluorescence threshold to eliminate diffuse signal. The same settings were used for all images. Colocalization spots were identified using the JaCoP plug-in (Bolte and Cordelières, 2006) for ImageJ (Manders' coefficient) and then manually counted.

Colocalization analysis for three channels was done on Airyscan images. Each channel was first processed by filtering and background subtraction to reduce noise. The same settings were used for all images of each channel. Images were segmented by thresholding in order to get binary masks that were finally combined using the image calculator tool (operation: AND) in order to obtain triple-colocalization masks. The latter were used to identify the number of mitochondria that show one site of colocalization with AnkB and phospho-Thr175/Thr186-SadA/B or Lamp1, respectively. 


\section{Statistical Analysis}

All plotted values are expressed as means \pm standard error of the mean (SEM). All experiments were performed independently at least three times. Specific numbers can be found in the figure legends. If not otherwise indicated, comparisons between two groups were performed using unpaired Students t-test, comparisons of more than two groups were done using one-way ANOVA followed by Tukey's or Dunnett's test with GraphPad Prism. For all analyses performed, significance was defined as ns: $p>0.05$; ${ }^{*} p \leq 0.05 ;{ }^{* *} p \leq 0.01 ;{ }^{* * *} p \leq 0.001 ;{ }^{* * *} p \leq 0.0001$. When not indicated, the comparison between conditions is not significantly different.

\section{Supplemental videos}

Video S1. Knockdown of SadA/B increases the mitochondrial motility and disturbs the balance of fission and fusion. Related to Figure $5 A$ and $5 B$.

Video S2. Knockdown of AnkB increases the mitochondrial motility and disturbs the balance of fission and fusion similar to SadA/B knockdown. Related to Figure 5C and 5D.

Video S3. Expression of Drp1-R376E increases the mitochondrial motility and disturbs the balance of fission and fusion in single axon neurons, similar to SadA/B and AnkB knockdown. Related to Figure 6E and 6G.

Video S4. LatA treatment of SadA/B knockdown neurons reduces the rate of fission and fusion and increases the percentage of fission events but does not restore mitochondrial motility. Related to Figure $8 \mathrm{C}$ and $8 \mathrm{D}$. 


\section{References}

Abrisch, R.G., Gumbin, S.C., Wisniewski, B.T., Lackner, L.L., and Voeltz, G.K. (2020). Fission and fusion machineries converge at ER contact sites to regulate mitochondrial morphology. J. Cell Biol. 219, e201911122.

Ambadipudi, S., Biernat, J., Riedel, D., Mandelkow, E., and Zweckstetter, M. (2017). Liquid-liquid phase separation of the microtubule-binding repeats of the Alzheimer-related protein Tau. Nat. Commun. 8, 1-13.

Ayalon, G., Hostettler, J.D., Hoffman, J., Kizhatil, K., Davis, J.Q., and Bennett, V. (2011). Ankyrin-B interactions with spectrin and dynactin-4 are required for dystrophin-based protection of skeletal muscle from exercise injury. J. Biol. Chem. 286, 7370-7378.

Barnes, A.P., Lilley, B.N., Pan, Y.A., Plummer, L.J., Powell, A.W., Raines, A.N., Sanes, J.R., and Polleux, F. (2007). LKB1 and SAD Kinases Define a Pathway Required for the Polarization of Cortical Neurons. Cell 129, 549-563.

Baum, T., and Gama, V. (2021). Dynamic properties of mitochondria during human corticogenesis. Development 148, dev194183.

Berthet, A., Margolis, E.B., Zhang, J., Hsieh, I., Zhang, J., Hnasko, T.S., Ahmad, J., Edwards, R.H., Sesaki, H., Huang, E.J., et al. (2014). Loss of mitochondrial fission depletes axonal mitochondria in midbrain dopamine neurons. J. Neurosci. 34, 14304-14317.

Biernat, J., Gustke, N., Drewes, G., Mandelkow, E., and Mandelkow, E. (1993). Phosphorylation of Ser262 strongly reduces binding of tau to microtubules: Distinction between PHF-like immunoreactivity and microtubule binding. Neuron 11, 153-163.

van der Bliek, A.M., Shen, Q., and Kawajiri, S. (2013). Mechanisms of mitochondrial fission and fusion. Cold Spring Harb. Perspect. Biol. 5, 011072.

Bolte, S., and Cordelières, F.P. (2006). A guided tour into subcellular colocalization analysis in light microscopy. J. Microsc. 224, 213-232.

Bright, N.J., Carling, D., and Thornton, C. (2008). Investigating the regulation of brain-specific kinases 1 and 2 by phosphorylation. J. Biol. Chem. 283, 14946-14954.

Britt, D.J., Farías, G.G., Guardia, C.M., and Bonifacino, J.S. (2016). Mechanisms of polarized organelle distribution in neurons. Front. Cell. Neurosci. 10, 1-7.

Cabrales Fontela, Y., Kadavath, H., Biernat, J., Riedel, D., Mandelkow, E., and Zweckstetter, M. (2017). Multivalent cross-linking of actin filaments and microtubules through the microtubuleassociated protein Tau. Nat. Commun. 8, 1-12.

Cagalinec, M., Safiulina, D., Liiv, M., Liiv, J., Choubey, V., Wareski, P., Veksler, V., and Kaasik, A. (2013). Principles of the mitochondrial fusion and fission cycle in neurons. J. Cell Sci. 126, 21872197.

Cavallini, A., Brewerton, S., Bell, A., Sargent, S., Glover, S., Hardy, C., Moore, R., Calley, J., Ramachandran, D., Poidinger, M., et al. (2013). An unbiased approach to identifying tau kinases 
that phosphorylate tau at sites associated with alzheimer disease. J. Biol. Chem. 288, 2333123347.

Chang, C., Shao, E., Mucke, L., Tau, A., Tau, N., and Tau, E. (2021). Tau: Enabler of diverse brain disorders and target of rapidly evolving therapeutic strategies. Science 371 , eabb8255.

Chang, D.T.W., Honick, A.S., and Reynolds, I.J. (2006). Mitochondrial trafficking to synapses in cultured primary cortical neurons. J. Neurosci. 26, 7035-7045.

Chaudhary, A.R., Berger, F., Berger, C.L., and Hendricks, A.G. (2018). Tau directs intracellular trafficking by regulating the forces exerted by kinesin and dynein teams. Traffic 19, 111-121.

Courchet, J., Lewis, T.L., Lee, S., Courchet, V., Liou, D.Y., Aizawa, S., and Polleux, F. (2013). Terminal axon branching is regulated by the LKB1-NUAK1 kinase pathway via presynaptic mitochondrial capture. Cell 153, 1510.

Courchet, V., Roberts, A.J., Meyer-Dilhet, G., Del Carmine, P., Lewis, T.L., Polleux, F., and Courchet, J. (2018). Haploinsufficiency of autism spectrum disorder candidate gene NUAK1 impairs cortical development and behavior in mice. Nat. Commun. 9.

Dhumale, P., Menon, S., Chiang, J., and Püschel, A.W. (2018). The loss of the kinases SadA and SadB results in early neuronal apoptosis and a reduced number of progenitors. PLoS One 13, 118.

Dixit, R., Ross, J.L., Goldman, Y.E., and Holzbaur, E.L.F. (2008). Differential Regulation of Dynein and Kinesin Motor Proteins by Tau. Science 319, 1086-1089.

Drewes, G., Trinczek, B., Illenberger, S., Biernat, J., Schmitt-Ulms, G., Meyer, H.E., Mandelkow, E.M., and Mandelkow, E. (1995). Microtubule-associated protein/microtubule affinity-regulating kinase $(\mathrm{p} 110$ (mark)). A novel protein kinase that regulates tau-microtubule interactions and dynamic instability by phosphorylation at the Alzheimer- specific site serine 262. J. Biol. Chem. 270, 7679-7688.

DuBoff, B., Götz, J., and Feany, M.B. (2012). Tau Promotes Neurodegeneration via DRP1 Mislocalization In Vivo. Neuron 75, 618-632.

Dupraz, S., Hilton, B.J., Husch, A., Santos, T.E., Coles, C.H., Stern, S., Brakebusch, C., and Bradke, F. (2019). RhoA Controls Axon Extension Independent of Specification in the Developing Brain. Curr. Biol. 29, 3874-3886.e9.

Ebneth, Godemann, R., Stamer, K., Illenberger, S., Trinczek, B., Mandelkow, E.-M., and Mandelkow, E. (2011). Overexpression of Tau Protein Inhibits Kinesin-dependent Trafficking of Vesicles, Mitochondria, and Endoplasmic Reticulum: Implications for Alzheimer's Disease. Cell 143, 777-794.

Elie, A., Prezel, E., Guérin, C., Denarier, E., Ramirez-Rios, S., Serre, L., Andrieux, A., FourestLieuvin, A., Blanchoin, L., and Arnal, I. (2015). Tau co-organizes dynamic microtubule and actin networks. Sci. Rep. 5, 1-10.

Ercan, E., Eid, S., Weber, C., Kowalski, A., Bichmann, M., Behrendt, A., Matthes, F., Krauss, S., 
Reinhardt, P., Fulle, S., et al. (2017). A validated antibody panel for the characterization of tau post-translational modifications. Mol. Neurodegener. 12, 1-19.

Fenton, A.R., Jongens, T.A., and Holzbaur, E.L.F. (2021). Mitochondrial dynamics: Shaping and remodeling an organelle network. Curr. Opin. Cell Biol. 68, 28-36.

Francy, C.A., Alvarez, F.J.D., Zhou, L., Ramachandran, R., and Mears, J.A. (2015). The mechanoenzymatic core of dynamin-related protein 1 comprises the minimal machinery required for membrane constriction. J. Biol. Chem. 290, 11692-11703.

Franker, M.A.M., and Hoogenraad, C.C. (2013). Microtubule-based transport -basic mechanisms, traffic rules and role in neurological pathogenesis. J. Cell Sci. 126, 2319-2329.

Fujiwara, I., Zweifel, M.E., Courtemanche, N., and Pollard, T.D. (2018). Latrunculin A Accelerates Actin Filament Depolymerization in Addition to Sequestering Actin Monomers. Curr. Biol. 28, 31833192.e2.

Fulga, T.A., Elson-schwab, I., Khurana, V., Steinhilb, M.L., Spires, T.L., Hyman, B.T., and Feany, M.B. (2007). Abnormal bundling and accumulation of F-actin mediates tau-induced neuronal degeneration in vivo. Nat. Cell Biol. 9, 139-148.

Galiano, M.R., Jha, S., Ho, T.S.Y., Zhang, C., Ogawa, Y., Chang, K.J., Stankewich, M.C., Mohler, P.J., and Rasband, M.N. (2012). A distal axonal cytoskeleton forms an intra-axonal boundary that controls axon initial segment assembly. Cell 149, 1125-1139.

Guo, Y., Li, D., Zhang, S., Yang, Y., Liu, J.J., Wang, X., Liu, C., Milkie, D.E., Moore, R.P., Tulu, U.S., et al. (2018). Visualizing Intracellular Organelle and Cytoskeletal Interactions at Nanoscale Resolution on Millisecond Timescales. Cell 175, 1430-1442.e17.

He, H.J., Wang, X.S., Pan, R., Wang, D.L., Liu, M.N., and He, R.Q. (2009). The proline-rich domain of tau plays a role in interactions with actin. BMC Cell Biol. 10, 1471-2121.

Hung, W., Hwang, C., Po, M.D., and Zhen, M. (2007). Neuronal polarity is regulated by a direct interaction between a scaffolding protein, Neurabin, and a presynaptic SAD-1 kinase in Caenorhabditis elegans. Development 134, 237-249.

Ji, W.K., Hatch, A.L., Merrill, R.A., Strack, S., and Higgs, H.N. (2015). Actin filaments target the oligomeric maturation of the dynamin GTPase Drp1 to mitochondrial fission sites. Elife 4, 1-25.

Kang, J.S., Tian, J.H., Pan, P.Y., Zald, P., Li, C., Deng, C., and Sheng, Z.H. (2008). Docking of Axonal Mitochondria by Syntaphilin Controls Their Mobility and Affects Short-Term Facilitation. Cell 132, 137-148.

Katoh, Y., Nozaki, S., Hartanto, D., Miyano, R., and Nakayama, K. (2015). Architectures of multisubunit complexes revealed by a visible immunoprecipitation assay using fluorescent fusion proteins. J. Cell Sci. 128, 2351-2362.

Kishi, M., Pan, Y.A., Crump, J.G., and Sanes, J.R. (2005). Mammalian SAD kinases are required for neuronal polarization. Science 307, 929-932.

Korobova, F., Ramabhadran, V., and Higgs, H.N. (2013). An Actin-Dependent Step in 
Mitochondrial Fission Mediated by the ER-Associated Formin INF2. Science 339, 464-468.

Korobova, F., Gauvin, T.J., and Higgs, H.N. (2014). A role for myosin II in mammalian mitochondrial fission. Curr. Biol. 24, 409-414.

Lee, K.S., Huh, S., Lee, S., Wu, Z., Kim, A.K., Kang, H.Y., and Lu, B. (2018). Altered ERmitochondria contact impacts mitochondria calcium homeostasis and contributes to neurodegeneration in vivo in disease models. PNAS 115, 8844-8853.

Lewis, T.L., Courchet, J., and Polleux, F. (2013). Cellular and molecular mechanisms underlying axon formation, growth, and branching. J. Cell Biol. 202, 837-848.

Lewis, T.L., Kwon, S.K., Lee, A., Shaw, R., and Polleux, F. (2018). MFF-dependent mitochondrial fission regulates presynaptic release and axon branching by limiting axonal mitochondria size. Nat. Commun. 9, 1-15.

Lilley, B.N., Pan, Y.A., and Sanes, J.R. (2013). SAD Kinases Sculpt Axonal Arbors of Sensory Neurons through Long- and Short-Term Responses to Neurotrophin Signals. Neuron 79, 39-53.

Lizcano, J.M., Göransson, O., Toth, R., Deak, M., Morrice, N.A., Boudeau, J., Hawley, S.A., Udd, L., Mäkelä, T.P., Hardie, D.G., et al. (2004). LKB1 is a master kinase that activates 13 kinases of the AMPK subfamily, including MARK/PAR-1. EMBO J. 23, 833-843.

Lorenzo, D.N., Badea, A., Davis, J., Hostettler, J., He, J., Zhong, G., Zhuang, X., and Bennett, V. (2014). A PIK3C3-Ankyrin-B-Dynactin pathway promotes axonal growth and multiorganelle transport. J. Cell Biol. 207, 735-752.

Losón, O.C., Song, Z., Chen, H., and Chan, D.C. (2013). Fis1, Mff, MiD49, and MiD51 mediate Drp1 recruitment in mitochondrial fission. Mol. Biol. Cell 24, 659-667.

Ma, H., Wu, J.X., Wang, J., Wang, Z.X., and Wu, J.W. (2016). Structure and inhibition analysis of the mouse SAD-B C-terminal fragment. Biosci. Biotechnol. Biochem. 80, 1939-1946.

Mangeol, P., Prevo, B., and Peterman, E.J.G. (2016). KymographClear and KymographDirect: Two tools for the automated quantitative analysis of molecular and cellular dynamics using kymographs. Mol. Biol. Cell 27, 1948-1957.

Matenia, D., Hempp, C., Timm, T., Eikhof, A., and Mandelkow, E.M. (2012). Microtubule Affinityregulating Kinase 2 (MARK2) turns on phosphatase and tensin homolog (PTEN)-induced kinase 1 (PINK1) at Thr-313, a mutation site in Parkinson disease: Effects on mitochondrial transport. J. Biol. Chem. 287, 8174-8186.

Monroy, B.Y., Sawyer, D.L., Ackermann, B.E., Borden, M.M., Tan, T.C., and Ori-Mckenney, K.M. (2018). Competition between microtubule-associated proteins directs motor transport. Nat. Commun. 9, 1-12.

Monroy, B.Y., Tan, T.C., Oclaman, J.M., Han, J.S., Simó, S., Niwa, S., Nowakowski, D.W., McKenney, R.J., and Ori-McKenney, K.M. (2020). A Combinatorial MAP Code Dictates Polarized Microtubule Transport. Dev. Cell 53, 60-72.e4.

Moore, A.S. and E.L.F.H. (2018). Mitochondrial-cytoskeletal interactions: dynamic associations that 
facilitate network function and remodeling. Curr Opin Physiol. 176, 94-100.

Moore, A.S., Wong, Y.C., Simpson, C.L., and Holzbaur, E.L.F. (2016). Dynamic actin cycling through mitochondrial subpopulations locally regulates the fission-fusion balance within mitochondrial networks. Nat. Commun. 7, 1-13.

Müller, M., Lutter, D., and Püschel, A.W. (2010). Persistence of the cell-cycle checkpoint kinase Wee1 in SadA- and SadB-deficient neurons disrupts neuronal polarity. J. Cell Sci. 123, 286-294.

Namba, T., Funahashi, Y., Nakamuta, S., Xu, C., Takano, T., and Kaibuchi, K. (2015). Extracellular and intracellular signaling for neuronal polarity. Physiol. Rev. 95, 995-1024.

Oeding, S.J., Majstrowicz, K., Hu, X.P., Schwarz, V., Freitag, A., Honnert, U., Nikolaus, P., and Bähler, M. (2018). Identification of Miro1 and Miro2 as mitochondrial receptors for myosin XIX. J. Cell Sci. 131.

Otera, H., Wang, C., Cleland, M.M., Setoguchi, K., Yokota, S., Youle, R.J., and Mihara, K. (2010). Mff is an essential factor for mitochondrial recruitment of Drp1 during mitochondrial fission in mammalian cells. J. Cell Biol. 191, 1141-1158.

Pathak, D., Sepp, K.J., and Hollenbeck, P.J. (2010). Evidence that myosin activity opposes microtubule-based axonal transport of mitochondria. J. Neurosci. 30, 8984-8992.

Qiang, L., Sun, X., Austin, T.O., Muralidharan, H., Jean, D.C., Liu, M., Yu, W., and Baas, P.W. (2018). Tau Does Not Stabilize Axonal Microtubules but Rather Enables Them to Have Long Labile Domains. Curr. Biol. 28, 2181-2189.e4.

Qu, F., Lorenzo, D.N., King, S.J., Brooks, R., Bear, J.E., and Bennett, V. (2016). Ankyrin-B is a PI3P effector that promotes polarized $\alpha 5 \beta 1$-integrin recycling via recruiting RabGAP1L to early endosomes. Elife 5, 1-25.

Rangaraju, V., Lauterbach, M., and Schuman, E.M. (2019). Spatially Stable Mitochondrial Compartments Fuel Local Translation during Plasticity. Cell 176, 73-84.e15.

Rodríguez-Asiain, A., Ruiz-Babot, G., Romero, W., Cubí, R., Erazo, T., Biondi, R.M., Bayascas, J.R., Aguilera, J., Gómez, N., Gil, C., et al. (2011). Brain Specific Kinase-1 BRSK1/SAD-B associates with lipid rafts: Modulation of kinase activity by lipid environment. Biochim. Biophys. Acta $1811,1124-1135$.

Saxton, W.M., and Hollenbeck, P.J. (2012). The axonal transport of mitochondria. J. Cell Sci. 125, 2095-2104.

Schelski, M., and Bradke, F. (2017). Neuronal polarization: From spatiotemporal signaling to cytoskeletal dynamics. Mol. Cell. Neurosci. 84, 11-28.

Seager, R., Lee, L., Henley, J.M., and Wilkinson, K.A. (2020). Mechanisms and roles of mitochondrial localisation and dynamics in neuronal function. Neuronal Signal. 4, 1-28.

Shelly, M., and Poo, M.M. (2011). Role of LKB1-SAD/MARK pathway in neuronal polarization. Dev. Neurobiol. 71, 508-527.

Sheng, Z.H., and Cai, Q. (2012). Mitochondrial transport in neurons: Impact on synaptic 
homeostasis and neurodegeneration. Nat. Rev. Neurosci. 13, 77-93.

Siahaan, V., Krattenmacher, J., Hyman, A.A., Diez, S., Hernández-Vega, A., Lansky, Z., and Braun, M. (2019). Kinetically distinct phases of tau on microtubules regulate kinesin motors and severing enzymes. Nat. Cell Biol. 21, 1086-1092.

Smirnova, E., Griparic, L., Shurland, D.-L., and Van Der Bliek, A.M. (2001). Drp1 Is Required for Mitochondrial Division in Mammalian Cells. Mol. Biol. Cell 12, 2245-2256.

Tan, R., Lam, A.J., Tan, T., Han, J., Nowakowski, D.W., Vershinin, M., Simó, S., Ori-McKenney, K.M., and McKenney, R.J. (2019). Microtubules gate tau condensation to spatially regulate microtubule functions. Nat. Cell Biol. 21, 1078-1085.

Tilokani, L., Nagashima, S., Paupe, V., and Prudent, J. (2018). Mitochondrial dynamics: Overview of molecular mechanisms. Essays Biochem. 62, 341-360.

Toyama, E.Q., Herzig, S., Courchet, J., Jr, T.L.L., Losón, O.C., Hellberg, K., Young, N.P., Chen, H., Polleux, F., Chan, D.C., et al. (2016). AMP-activated protein kinase mediates mitochondrial fission in response to energy stress. Science 351, 275-282.

Veleva-Rotse, B.O., Smart, J.L., Baas, A.F., Edmonds, B., Zhao, Z. ming, Brown, A., Klug, L.R., Hansen, K., Reilly, G., Gardner, A.P., et al. (2014). STRAD pseudokinases regulate axogenesis and LKB1 stability. Neural Dev. 9, 5.

Vershinin, M., Carter, B.C., Razafsky, D.S., King, S.J., and Gross, S.P. (2007). Multiple-motor based transport and its regulation by Tau. Proc. Natl. Acad. Sci. U. S. A. 104, 87-92.

Wegmann, S., Eftekharzadeh, B., Tepper, K., Zoltowska, K.M., Bennett, R.E., Dujardin, S., Laskowski, P.R., MacKenzie, D., Kamath, T., Commins, C., et al. (2018). Tau protein liquid-liquid phase separation can initiate tau aggregation. EMBO J. 37, 1-21.

Witte, H., Neukirchen, D., and Bradke, F. (2008). Microtubule stabilization specifies initial neuronal polarization. J. Cell Biol. 180, 619-632.

Wong, Y.C., Ysselstein, D., and Krainc, D. (2018). Mitochondria-lysosome contacts regulate mitochondrial fission via RAB7 GTP hydrolysis. Nature 554, 382-386.

Wong, Y.C., Peng, W., Wong, Y.C., Peng, W., and Krainc, D. (2019). Lysosomal Regulation of Inter-mitochondrial Contact Fate and Motility in Charcot-Marie-Tooth Type 2. Dev. Cell 50, 339354.e4.

Wu, J.-X., Cheng, Y.-S., Wang, J., Chen, L., Ding, M., and Wu, J.-W. (2015). Structural insight into the mechanism of synergistic autoinhibition of SAD kinases. Nat. Commun. 6, 8953.

Yang, Z., Xue, B., Umitsu, M., Ikura, M., Muthuswamy, S.K., and Neel, B.G. (2012). The Signaling Adaptor Gab1 Regulates Cell Polarity by Acting as a PAR Protein Scaffold. Mol. Cell 47, 469-483. Yoshida, H., and Goedert, M. (2012). Phosphorylation of microtubule-associated protein tau by AMPK-related kinases. J. Neurochem. 120, 165-176.

Yoshimori, T., lijima, M., and Sesaki, H. (2018). A brain-enriched Drp1 isoform associates with lysosomes, late endosomes, and the plasma membrane. J. Biol. Chem. 293, 11809-11822. 


\section{FIGURE 1}

A

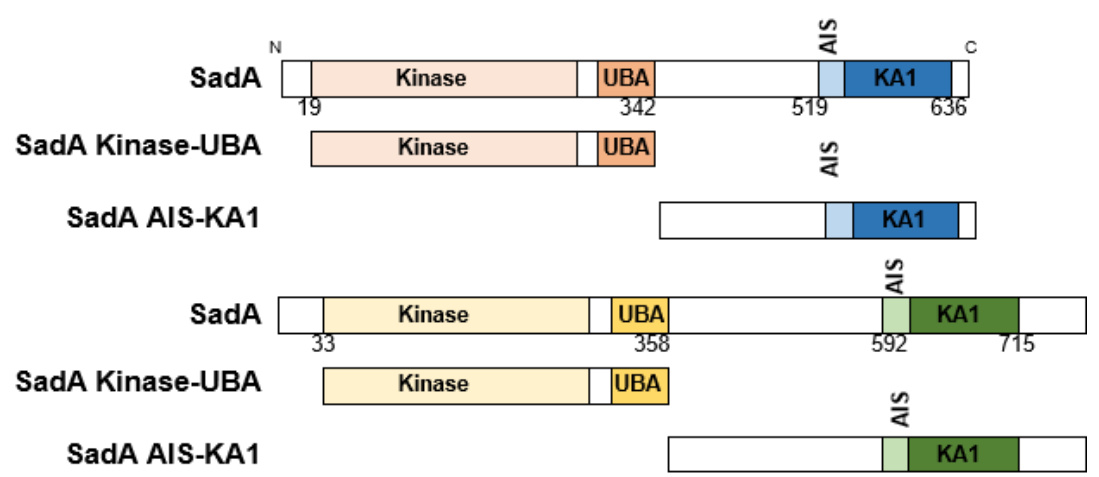

B

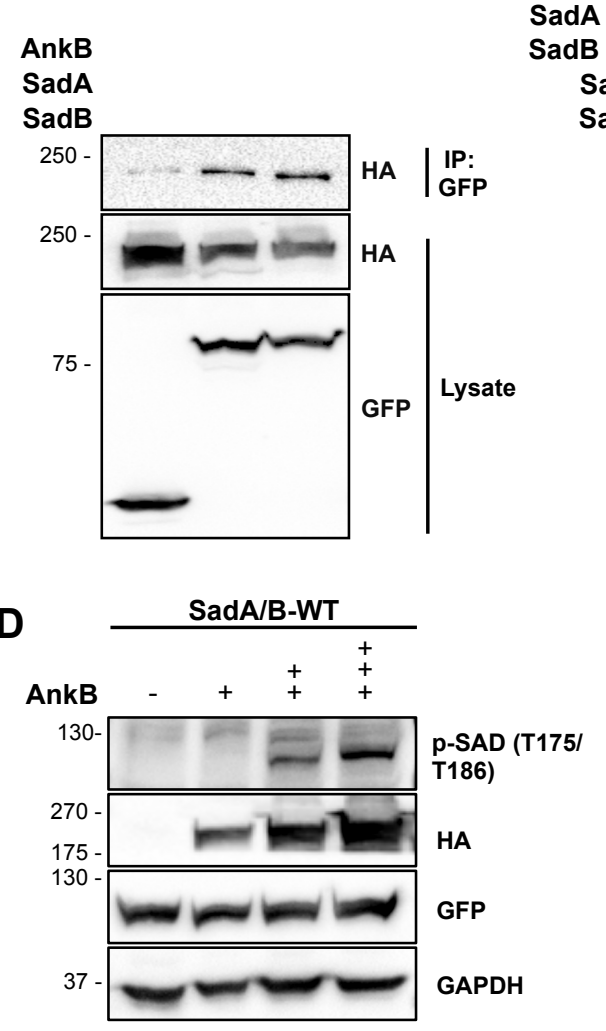

C
AnkB

adA Kinase-UBA

Binase-UBA

SadB AIS-KA1
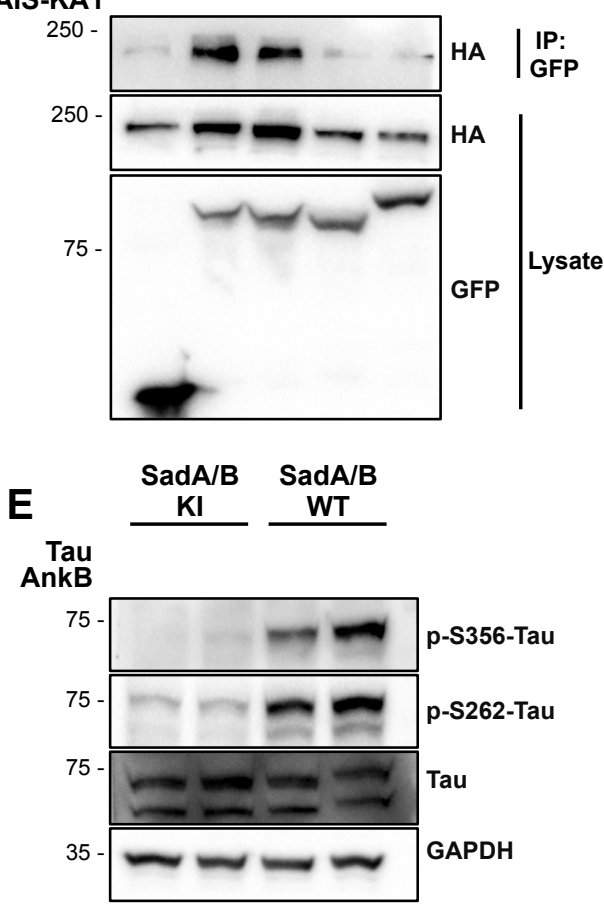


\section{FIGURE 2}

A
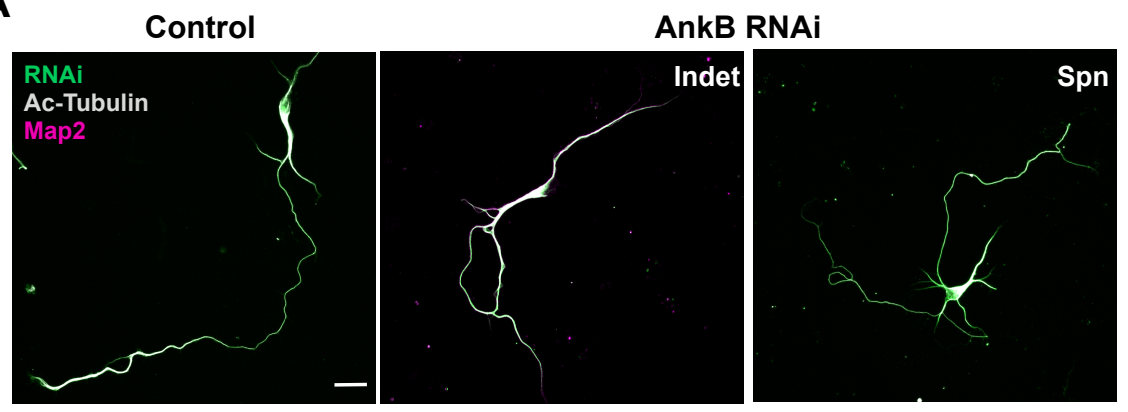

C

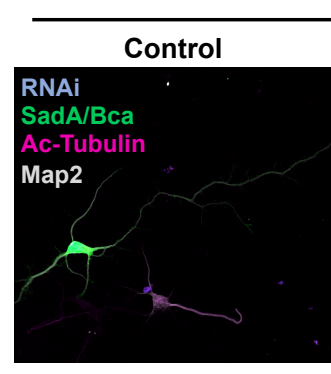

GFP
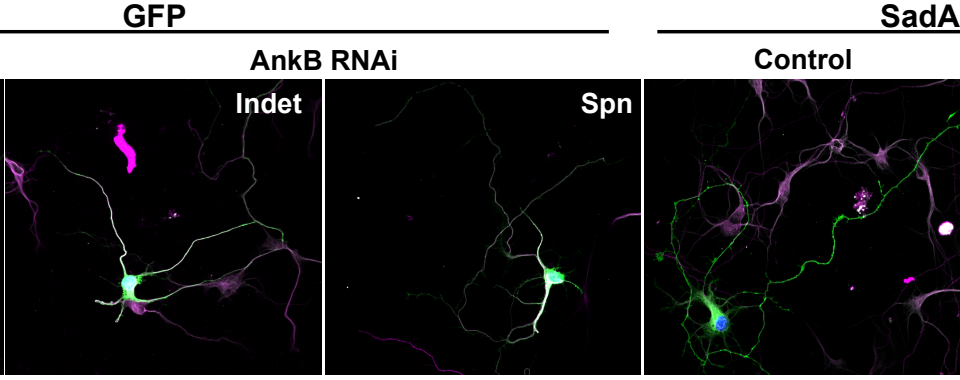

B

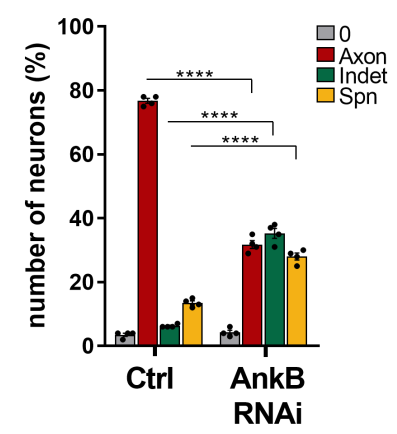

AnkB RNAi

D

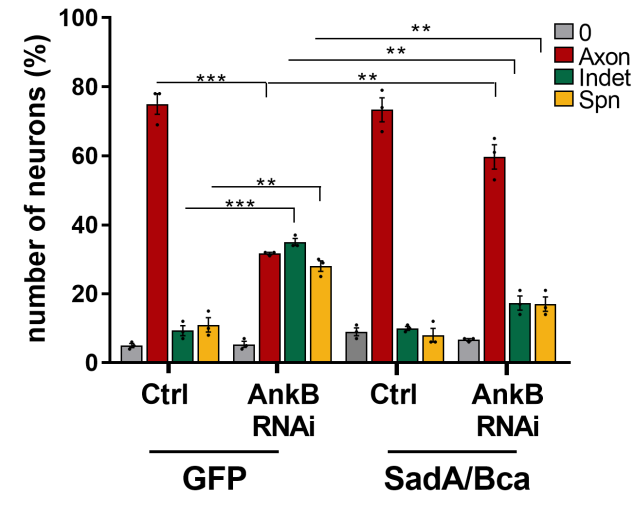




\section{FIGURE 3}
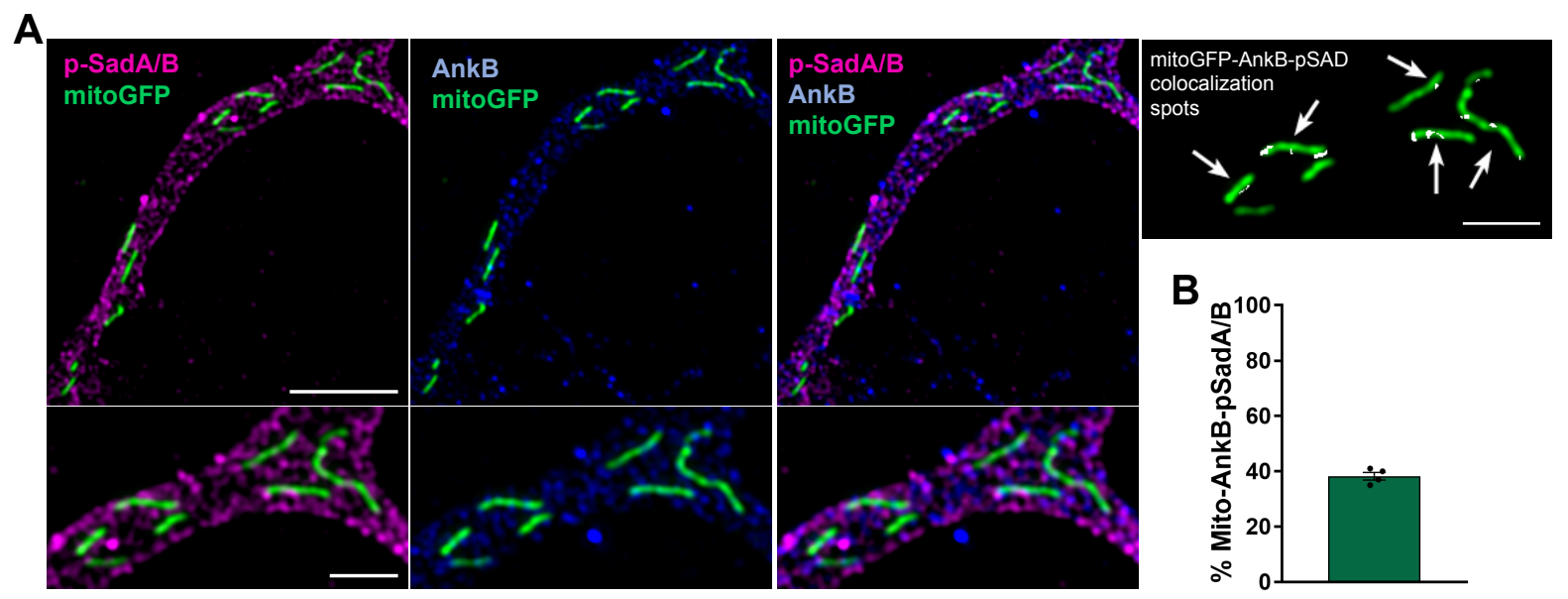

C
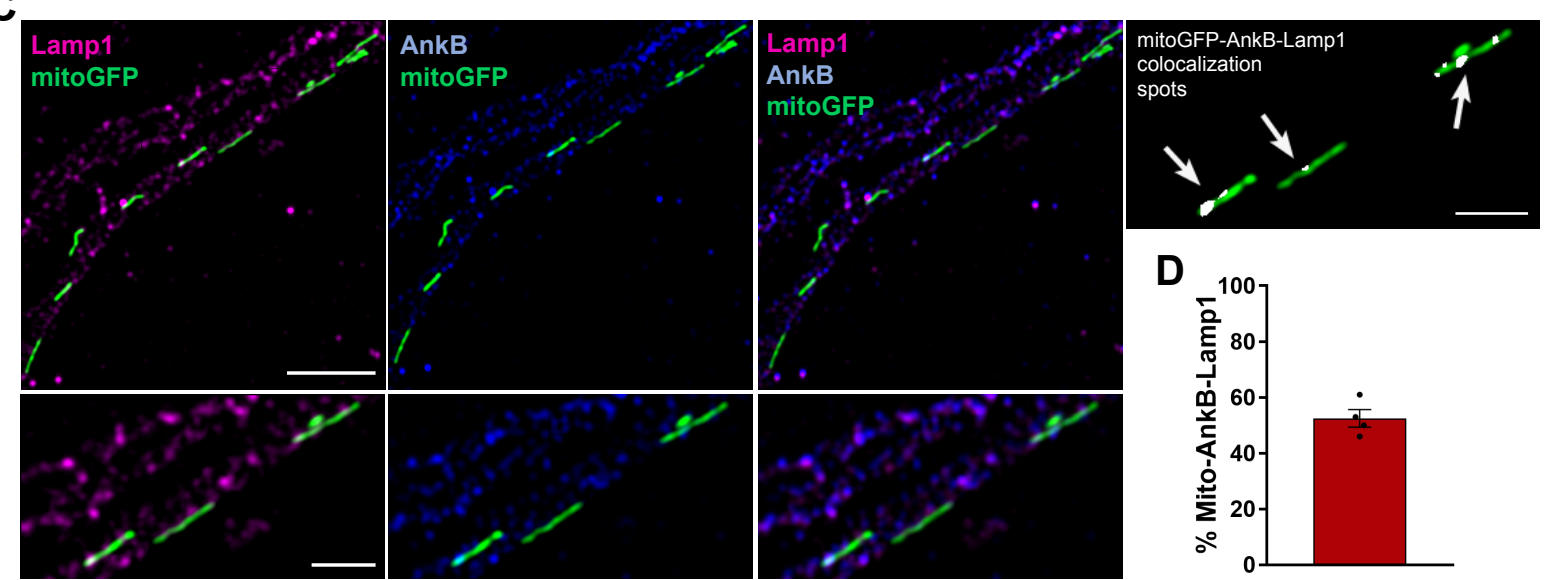

$\mathbf{E}$
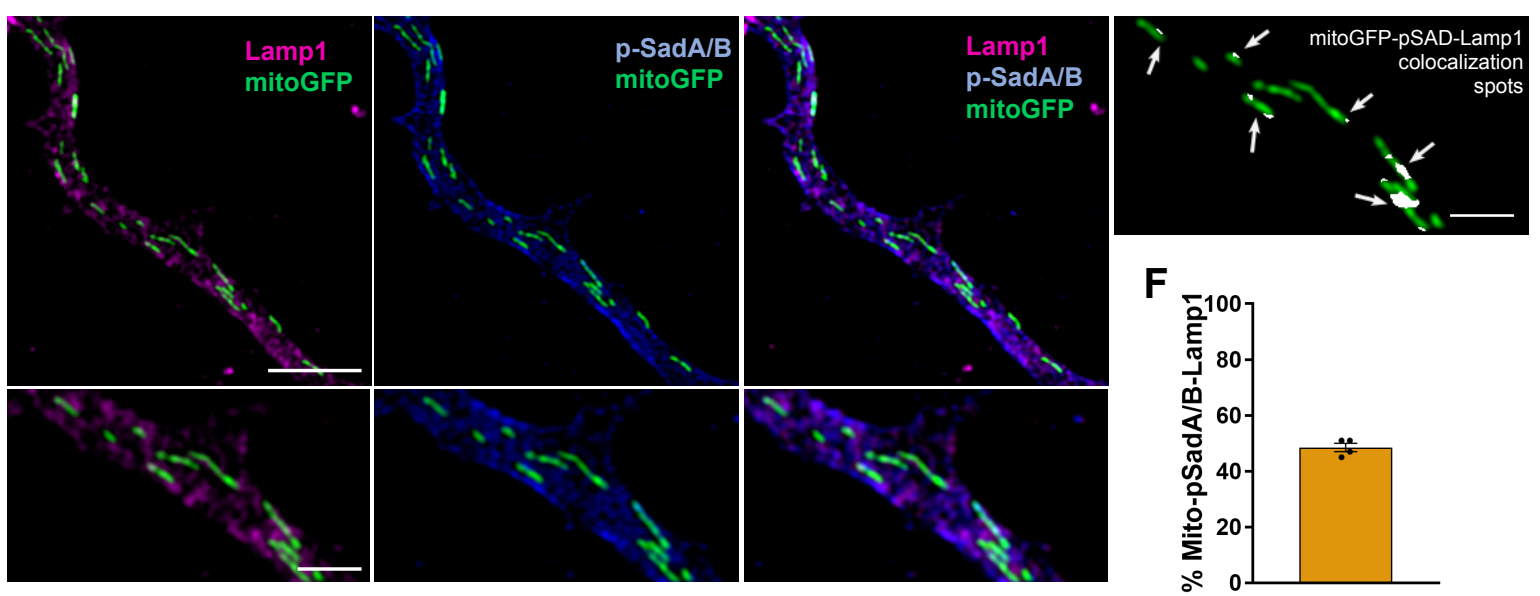


\section{FIGURE 4}
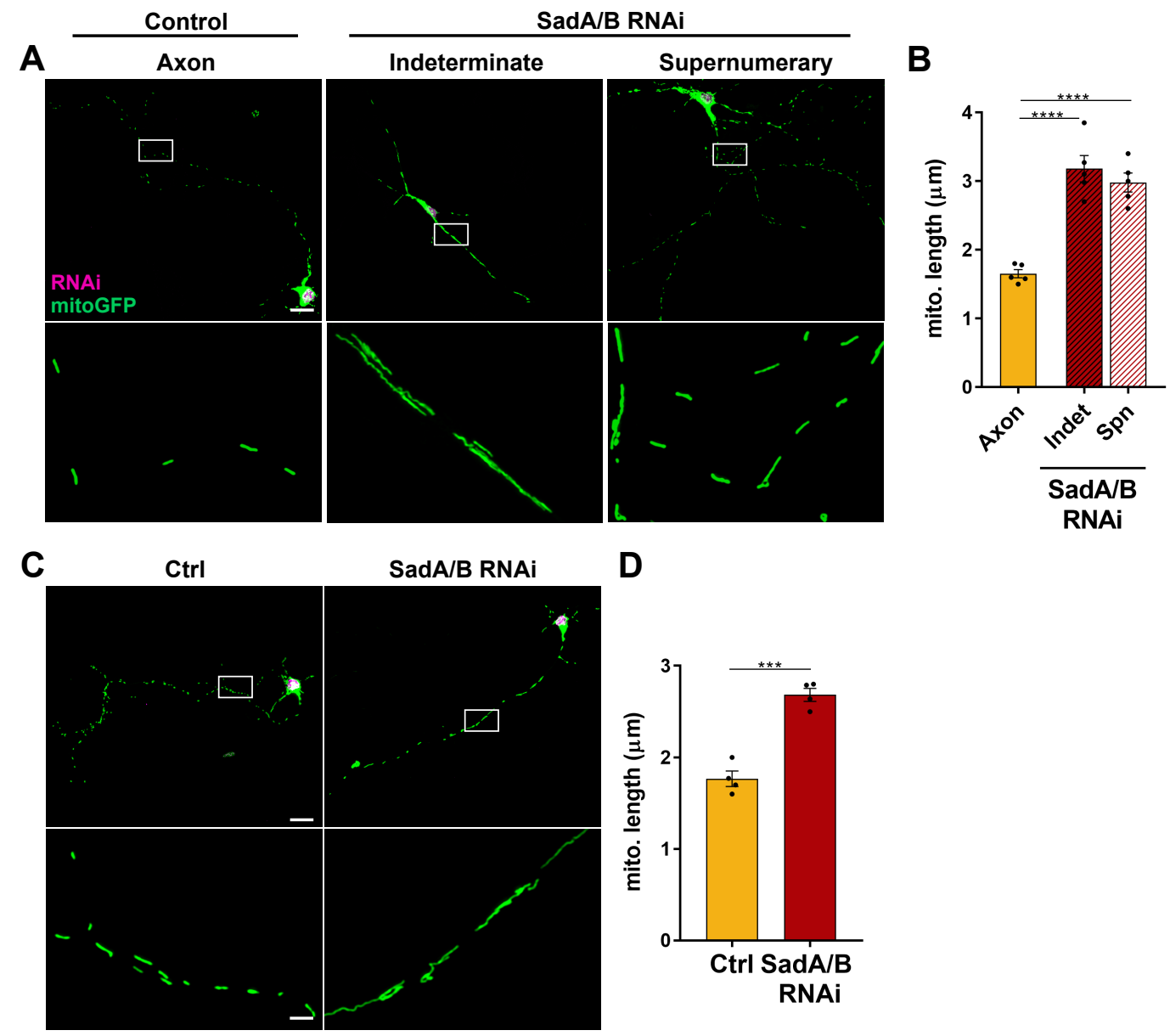

D
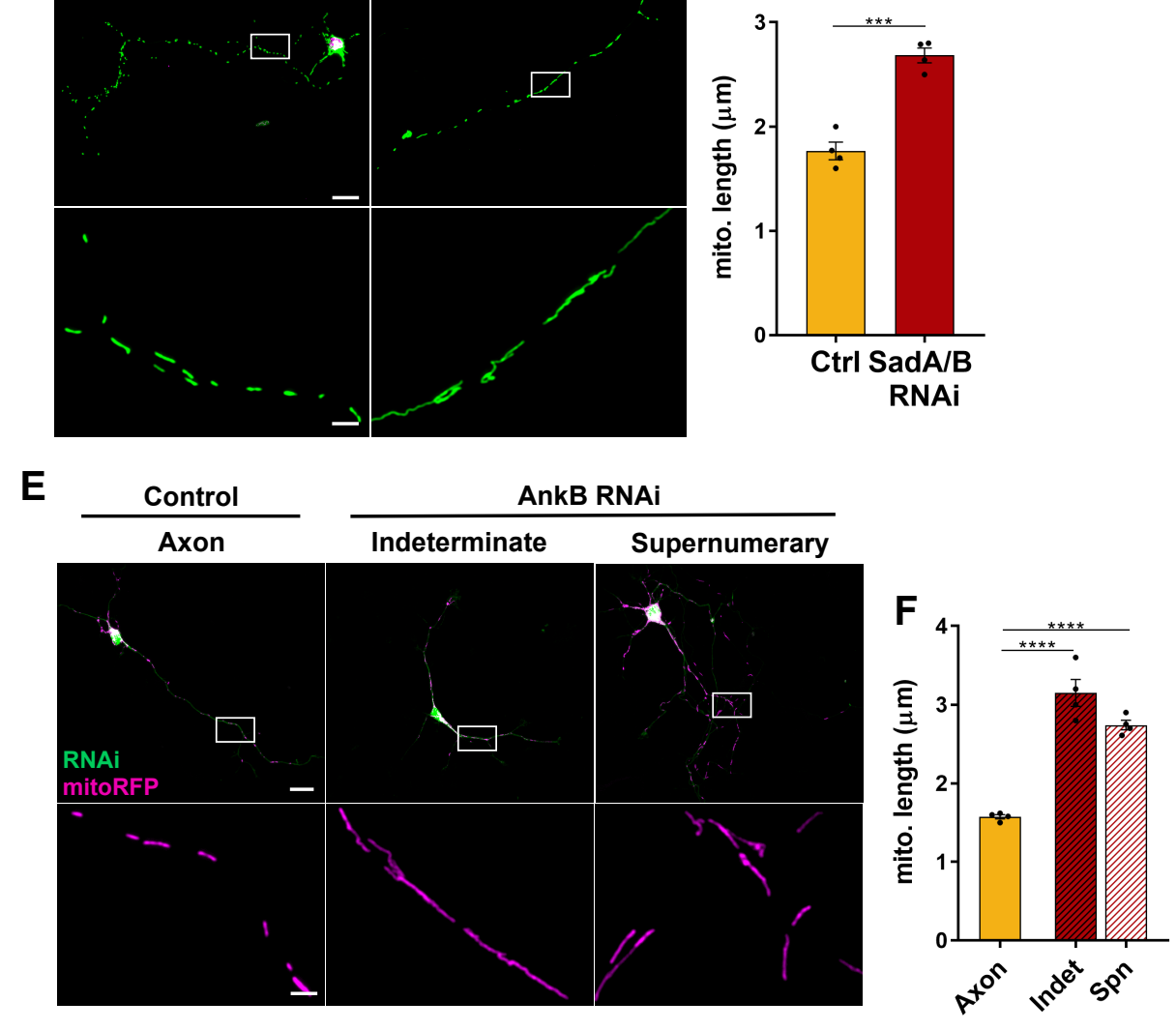

AnkB RNA

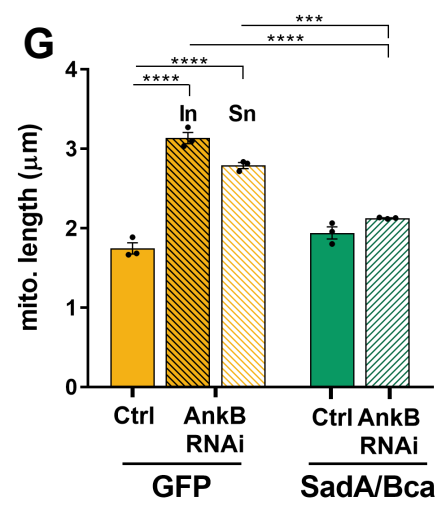




\section{FIGURE 5}

A
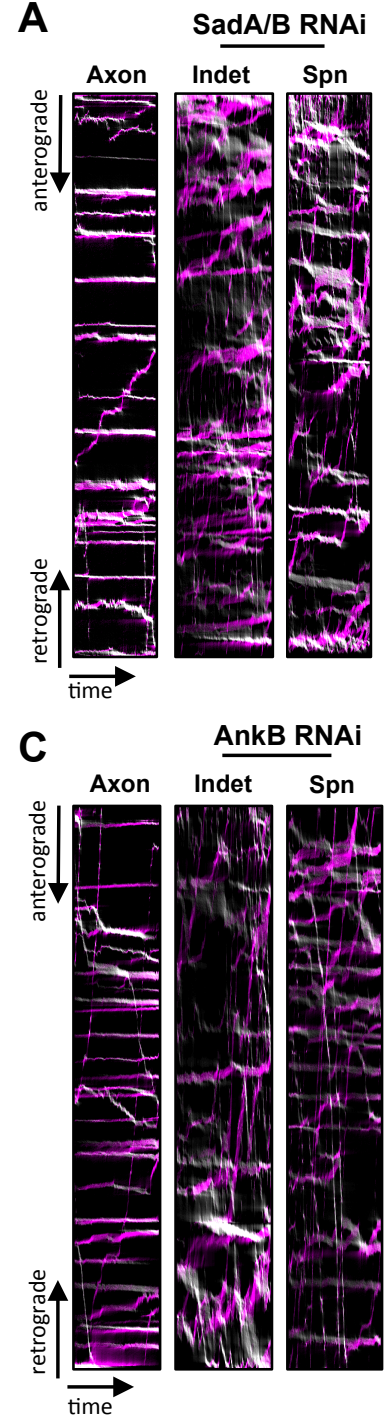

E

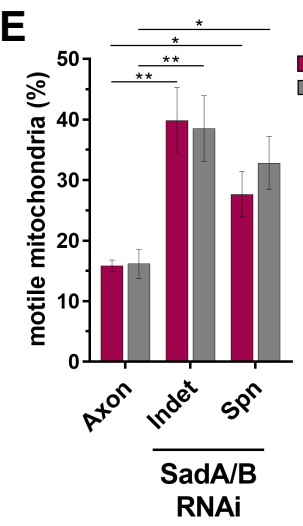

$F$
B 0 min

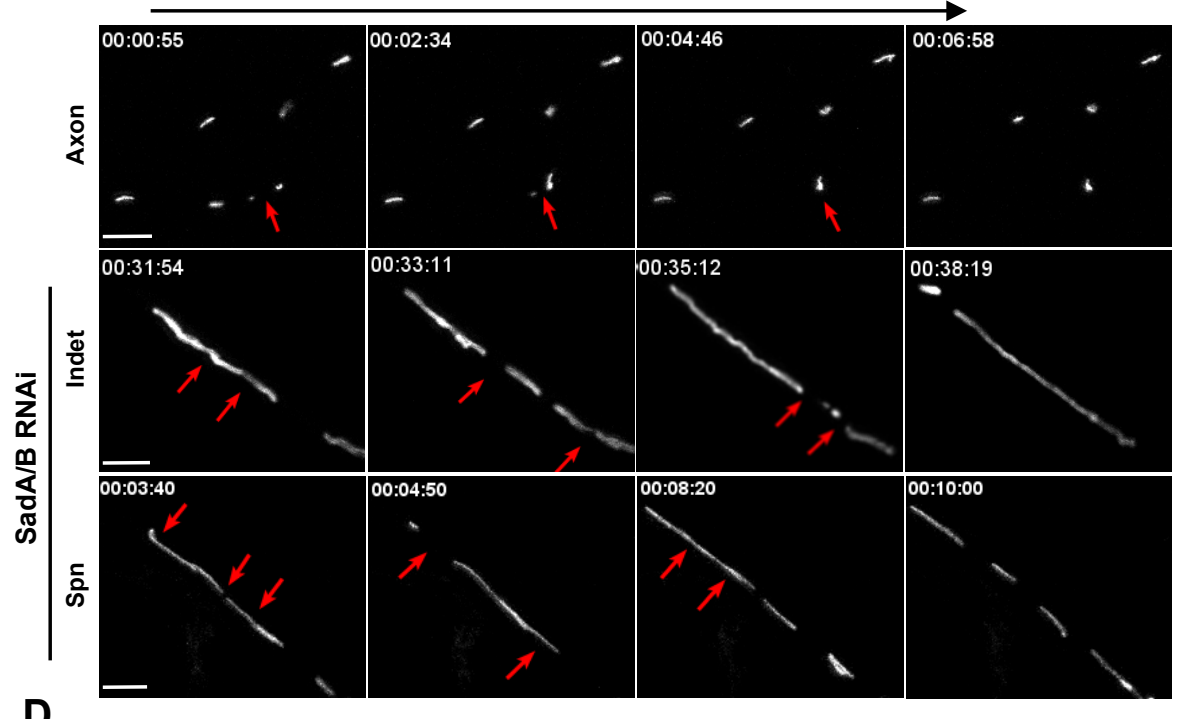

D
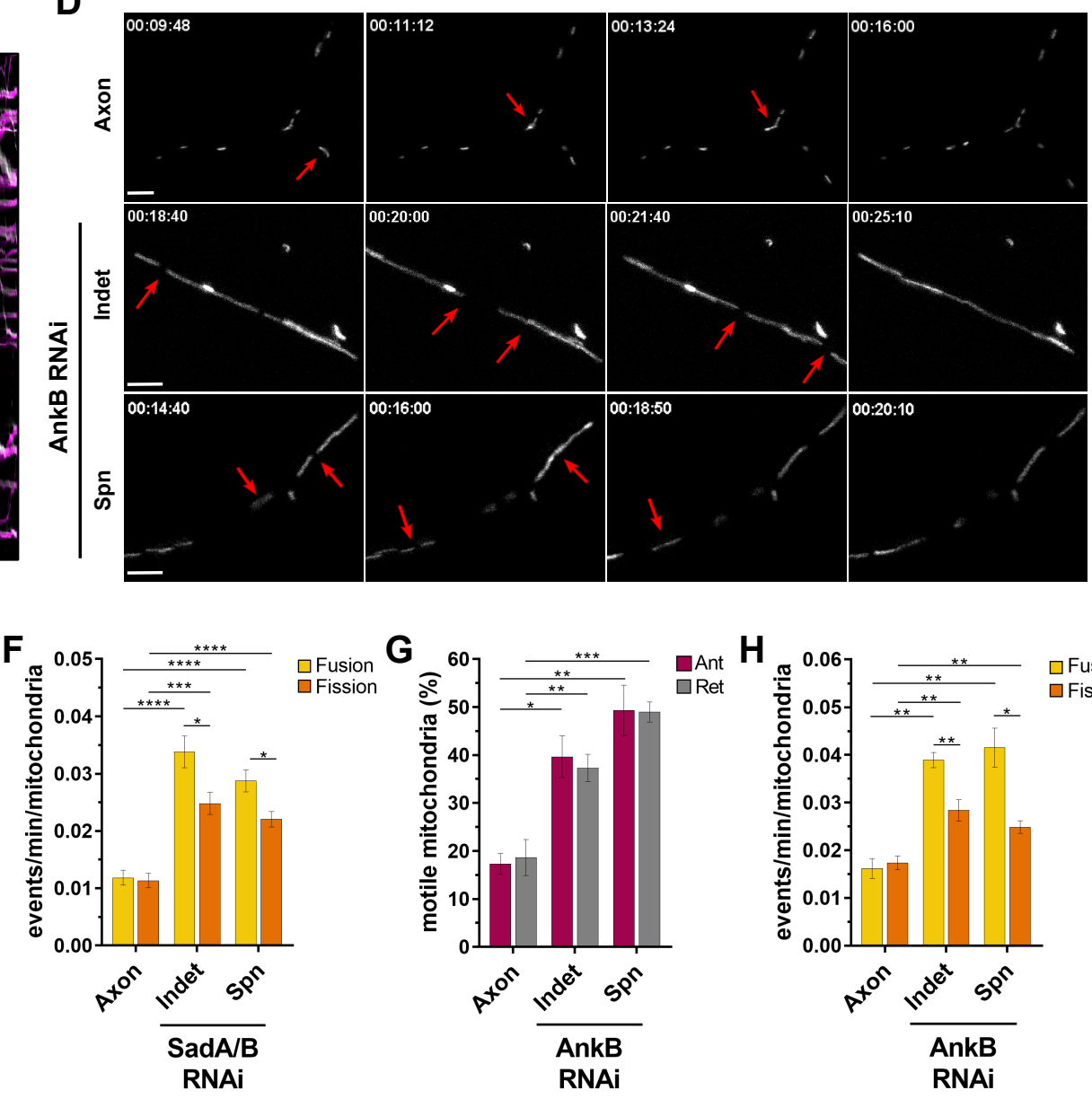

$\mathbf{G}$

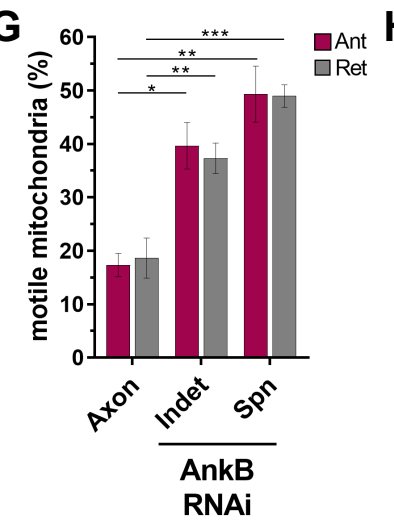

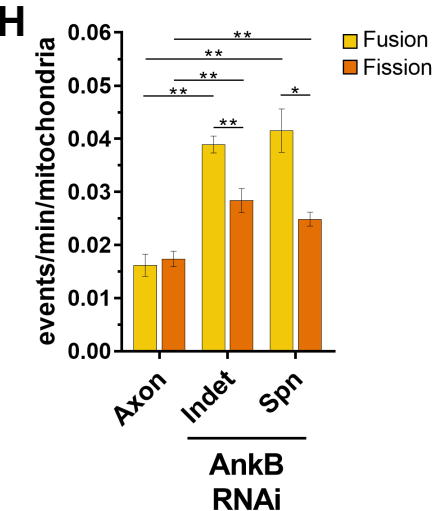




\section{FIGURE 6}
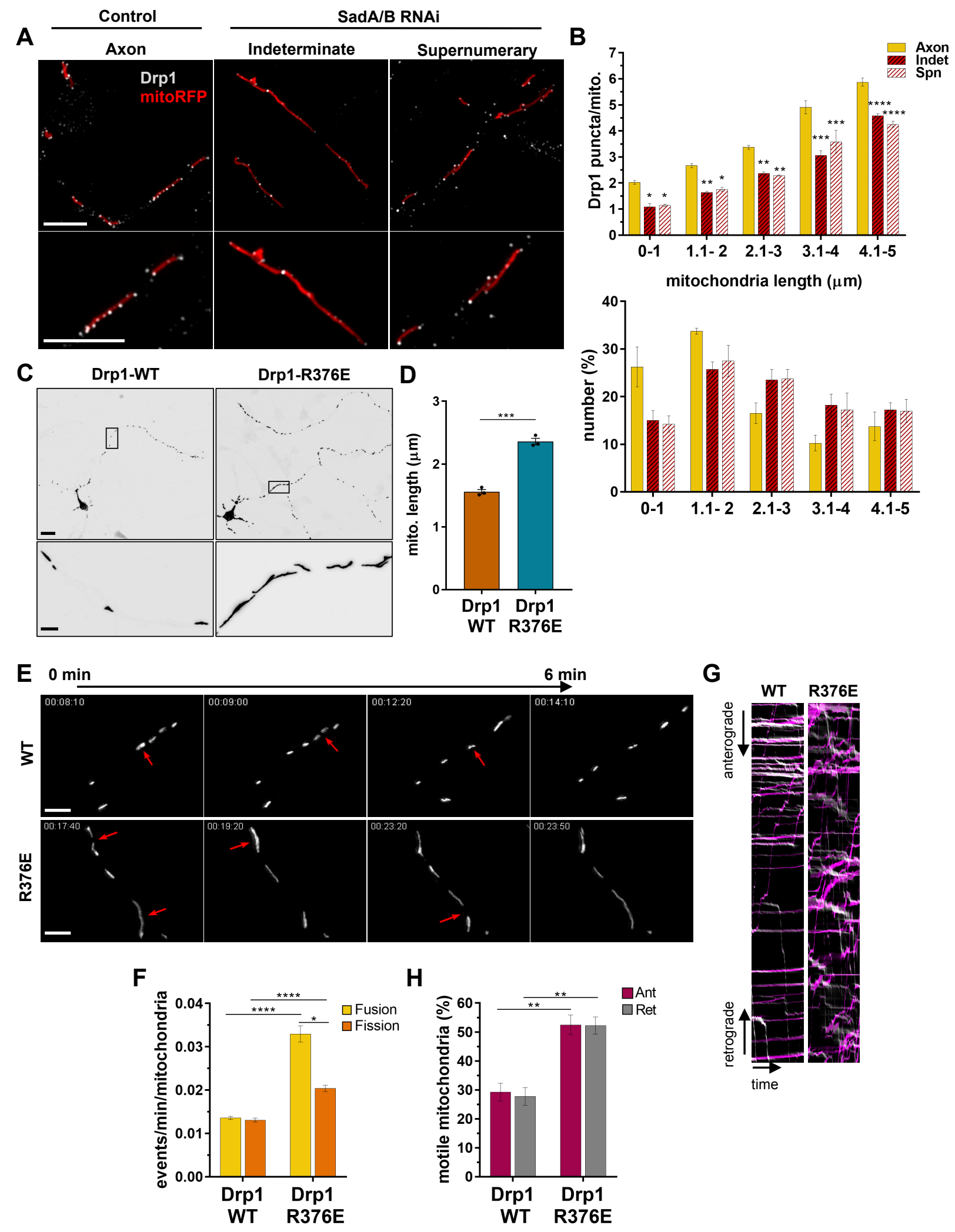


\section{FIGURE 7}

A

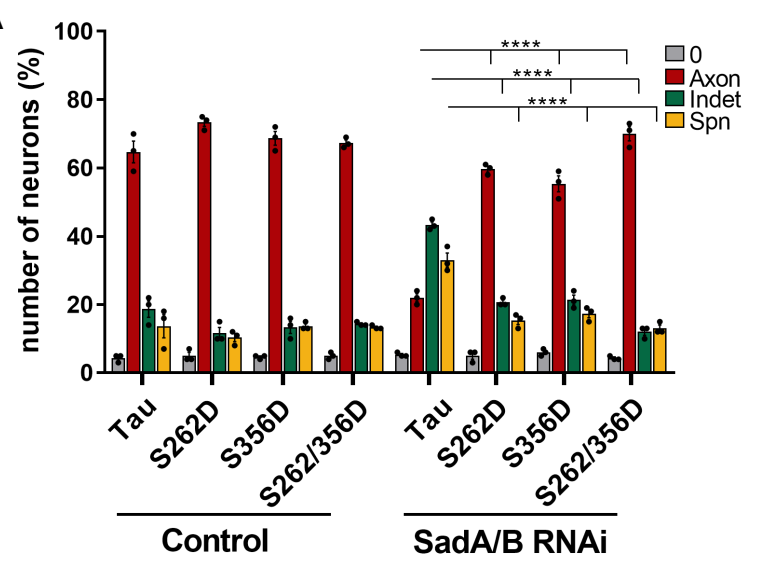

B

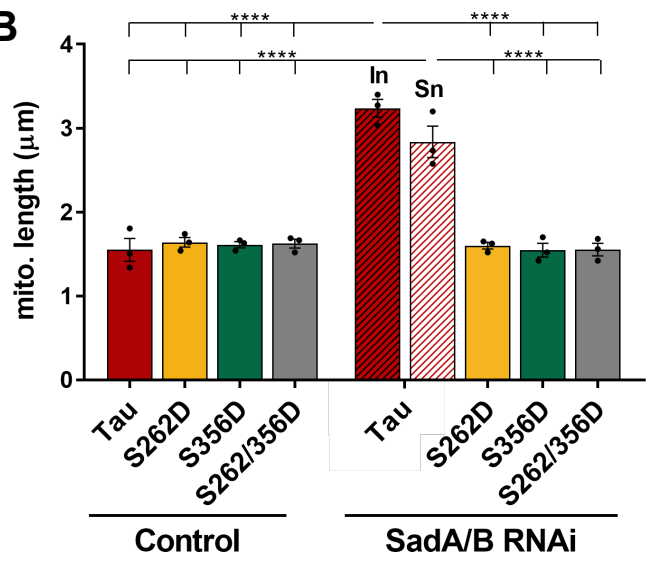

C

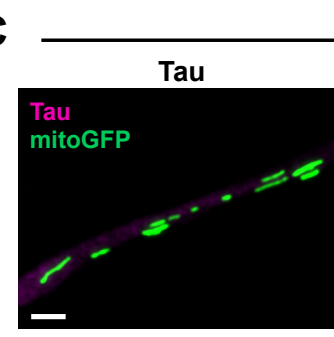

Control

S262D S356D

S262/S356D
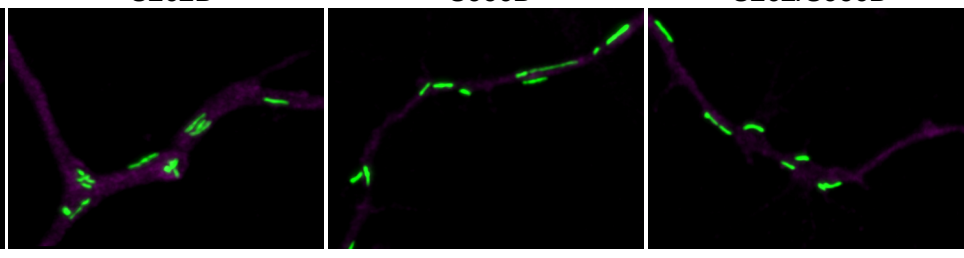

SadA/B RNAi

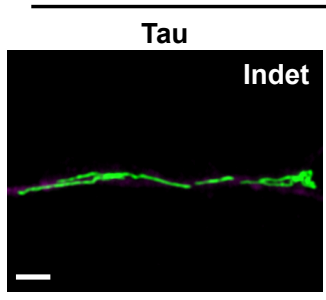

Tau

S262D
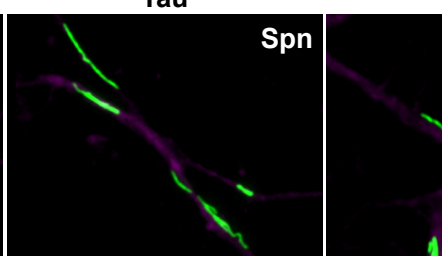

D
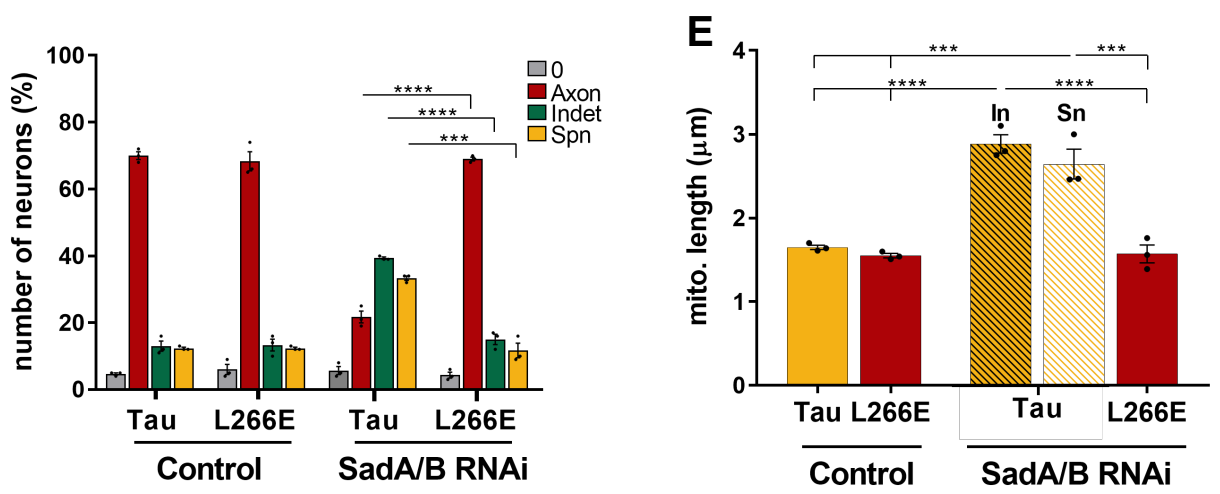


\section{FIGURE 8}

A

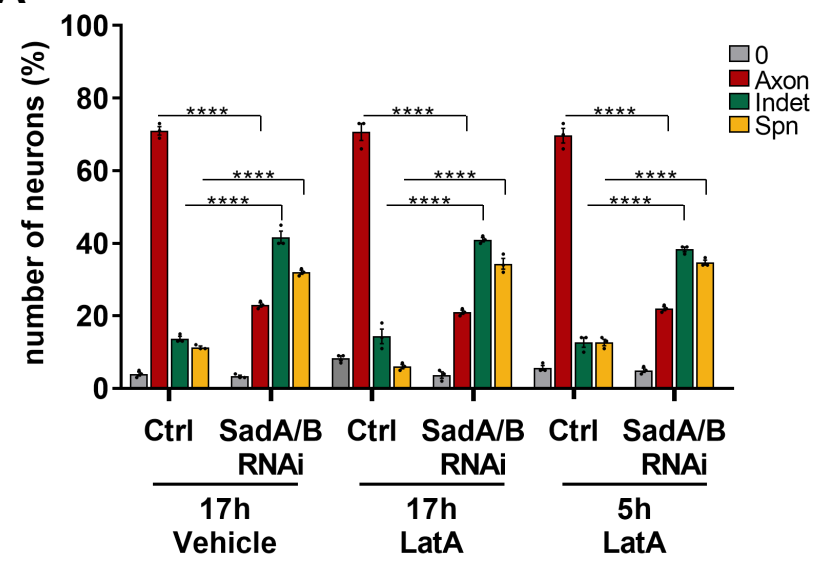

B

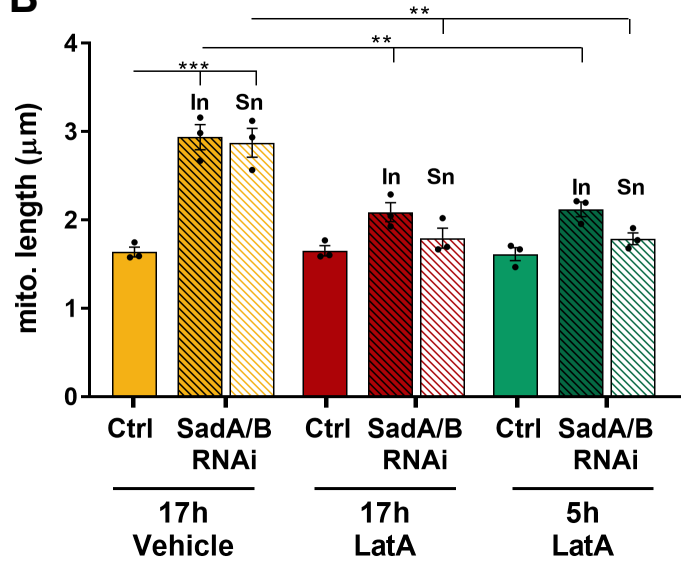

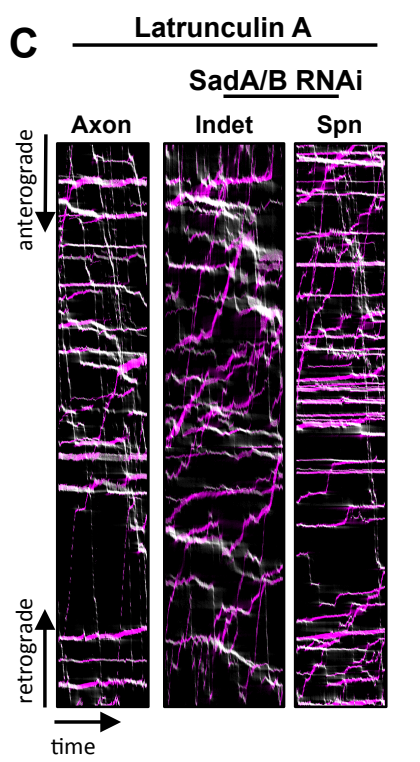

D 0 min $\underset{\text { Latrunculin A }}{\stackrel{6}{\longrightarrow}}$
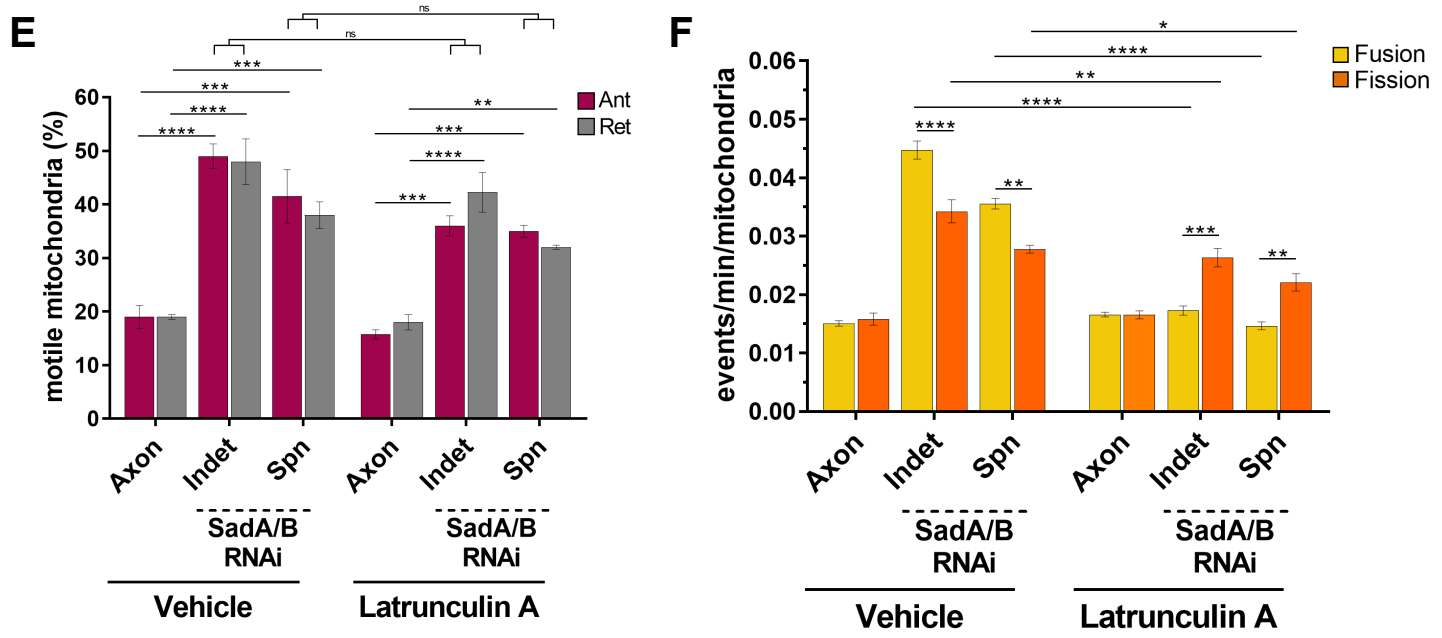